\title{
EXACT AND APPROXIMATE ALGORITHMS FOR THE LONGEST INDUCED PATH PROBLEM
}

\author{
Ruslán G. Marzo and Celso C. Ribeiro*
}

\begin{abstract}
The longest induced path problem consists in finding a maximum subset of vertices of a graph such that it induces a simple path. We propose a new exact enumerative algorithm that solves problems with up to 138 vertices and 493 edges and a heuristic for larger problems. Detailed computational experiments compare the results obtained by the new algorithms with other approaches in the literature and investigate the characteristics of the optimal solutions.
\end{abstract}

Mathematics Subject Classification. 05C30, 05C38, 05C85, 68W25, 90C27, 90C35.

Received October 16, 2020. Accepted January 14, 2021.

\section{INTRODUCTION}

Let $G=(V, E)$ be an undirected graph defined by its set of $n$ vertices $V=\{1, \cdots, n\}$ and its edge set $E \subseteq V \times V$. For any subset of vertices $S \subseteq V$, let $G[S]=\left(S, E^{\prime}\right)$ denote the subgraph induced by $S$ in $G$, where $E^{\prime}$ contains all edges from $E$ that have both of their endpoints in $S$. The longest induced path problem (LIPP) is defined as that of finding the subset $S \subseteq V$ of largest cardinality such that the resulting induced subgraph, $G[S]$, is a simple path [13].

The longest induced path problem has applications in various network analysis and design contexts. The graph diameter, which is defined as the length of the longest shortest path in a graph, is often used to quantify graph communication properties. In particular, the graph diameter provides an intuitive measure of the worstcase pairwise distance, e.g., the longest of the shortest communication paths in a communication network or the longest of the shortest routes in a transportation network. The longest induced path can also be considered as a measure of the worst-case pairwise distance in other practical scenarios, where vertices can either fail, be overloaded or be destroyed by an adversary (depending on the application context) and thus cannot be used in any communication or transportation path. In these cases, some subset of vertices cannot transmit a message in a communication network or cannot serve as a transshipment point in a transportation network and, consequently, detours or alternative shortest paths must be used. In other words, the objective of the longest induced path problem is to identify the worst possible case for the shortest distance between any two vertices in the graph, given that these vertices remain connected by some path while the rest of the vertices may fail [13].

The longest induced path problem is also known in the literature as the maximum induced path problem (see e.g. $[2,7]$ ). The maximum weighted induced path problem has applications in large communication and

Keywords. Longest induced path problem, exact algorithm, backtracking, heuristics.

Institute of Computing, Universidade Federal Fluminense, 24210-346 Niterói, RJ, Brazil.

*Corresponding author: celso@ic.uff.br 
neural networks when the worst case communication time needs to be evaluated [7]. Furthermore, LIPP is related to the snake-in-the-box problem, which was described by Kautz [11] and has applications in the theory of error-correcting codes. It consists in finding the longest possible induced path along the edges of a hypercube graph.

Another interesting interpretation of LIPP appears in social networks with information cascades. Matsypura et al. [13] observed that an optimal solution of the longest induced path problem in this context provides the longest possible path of information transmission, where one end vertex of the path corresponds to the seed of the information cascade and the other end vertex is the last to learn this piece of information.

Even though the graph diameter is computable in polynomial time [1], the decision version of the longest induced path problem is NP-complete ([6], p. 196). The problem remains NP-complete even for bipartite graphs. Finding the longest simple path (not necessarily induced) is also NP-complete. Polynomially solvable classes of the problem for specific graph families are discussed e.g. in [7-10,12]. Bergougnoux and Kanté [4] designed a framework to obtain efficient algorithms for several problems with a global constraint (acyclicity or connectivity), including the longest induced path.

Although the longest induced path problem belongs to an interesting class of network optimization problems with practical applications in various network contexts, the literature on solution approaches for this problem is rather limited, possibly due to its inherent computational complexity. Matsypura et al. [13] were the first to develop exact solution approaches for the longest induced path problem for general graphs, based on integer programming (IP) techniques. They proposed four IP formulations based on three conceptually different interpretations of the problem. In addition, they provided an exact iterative algorithm that solves a sequence of smaller MIPs and developed a randomized heuristic to find induced paths in large networks. Bökler et al. [5] proposed stronger integer linear programming formulations using cut (or generalized subtour elimination) constraints and clique inequalities.

In this work, we focus on solving the longest induced path problem for different classes of general graphs. We propose an exact enumerative algorithm and a heuristic for the problem. We review in Section 2 the existing solutions approaches proposed by Matsypura et al. [13] and Bökler et al. [5], from where we take the same test instances that will be used in our computational experiments. Section 3 describes the proposed exact enumerative algorithm and a new heuristic. In Section 4 we present computational experiments to assess the performance of the proposed solution methods. Section 5 investigates the correlation of the running times of the proposed exact algorithm and the sizes of the longest induced paths with some characteristics of the test instances. We also analyze the eccentricity and the degree of the extreme vertices of the optimal solutions obtained with this exact algorithm. Concluding remarks are drawn in Section 6.

\section{EXISTING APPROACHES}

In this section, we first summarize two integer programming formulations for the longest induced path problem (LIPP) developed by Matsypura et al. [13], referred to as IP3 and IP3c. The key idea behind these formulations is first to model a walk in the underlying graph and then force it to be an induced path by using additional constraints.

We assume that a walk in a graph starts at some vertex at time $t=0$. Then, at time $t=1$, it visits one of its neighbors. The walk continues visiting other vertices until time $t=T$ or no further moves are possible. The value of $T$ can be set to $|V|-1$ unless a better upper bound on the size of the longest induced path is available.

Both models IP3 and IP3c make use of the same binary variable $x_{i}^{t}$ associated to each vertex $i \in V$ of the graph and each time $t \in\{0, \cdots, T\}$ :

$$
x_{i}^{t}= \begin{cases}1, & \text { if and only if vertex } i \text { is visited at time } t \\ 0, & \text { otherwise. }\end{cases}
$$

A new vertex is visited at each time period whenever possible. If the walk cannot be continued at time $t=\tau$, then $x_{i}^{t}=0$ for all $i \in V, t \geq \tau$. To ensure that the walk is an induced path, it is necessary to verify that there 
are no shortcuts to the previously visited vertices and that no vertex that is not a neighbor of the current vertex can be visited at the next step. Based on these considerations, formulation IP3 is:

$$
\begin{aligned}
& \text { (IP3): } \quad \max \sum_{t=0}^{T} \sum_{i \in V} x_{i}^{t} \\
& \text { subject to: } \\
& \begin{array}{ll}
\sum_{i \in V} x_{i}^{t} \leq 1, & \forall t \in\{0, \cdots, T\}, \\
\sum_{t=0}^{T} x_{i}^{t} \leq 1, & \forall i \in V, \\
x_{i}^{t}+x_{j}^{t+1} \leq 1, & \forall(i, j) \notin E, \forall t \in\{0, \cdots, T-1\}, \\
x_{i}^{t}+x_{j}^{\tau} \leq 1, & \forall(i, j) \in E, \forall t \in\{0, \cdots, T-2\}, \forall \tau \in\{t+2, \cdots, T\}, \\
\sum_{i \in V} x_{i}^{t} \leq \sum_{i \in V} x_{i}^{t-1}, & \forall t \in\{1, \cdots, T\}, \\
x_{i}^{t} \in\{0,1\}, & \forall i \in V, \forall t \in\{0, \cdots, T\} .
\end{array}
\end{aligned}
$$

The objective function (2.1) maximizes the number of vertices in the resulting walk. Constraints (2.2) enforce that only one vertex can be visited at any time. Constraints (2.3) ensure that the walk cannot visit any vertex twice. Constraints (2.4) state that the walk can traverse only existing edges in E. Constraints (2.5) ensure that there are no shortcuts in a walk, i.e., the walk is, in fact, an induced path. Constraints (2.6) enforce that the walk cannot be interrupted, i.e., if the walk stops at time $t-2$ and no vertices are visited at time $t-1$, then it cannot start again from another vertex at time $t$. Finally, constraints (2.7) represent the integrality requirements.

Formulation IP3 has $O(T|V|)$ variables and $O\left(T|V|^{2}+T^{2}|E|\right)$ constraints. The number of constraints can be reduced by aggregating constraints (2.4) and (2.5), resulting in a more compact formulation denoted by IP3c with the same LP bound; see ([13], Sect. 3). Specifically, constraints (2.4) can be aggregated as:

$$
\sum_{j \in V:(i, j) \notin E} x_{j}^{t+1} \leq 1-x_{i}^{t}, \quad \forall i \in V, \forall t \in\{0, \cdots, T-1\},
$$

meaning that if vertex $i$ is visited at time $t$, i.e., $x_{i}^{t}=1$, then all vertices non-adjacent to $i$, i.e., those in $\{j \in V \mid(i, j) \notin E\}$, cannot be visited at time $t+1$. On the other hand, if $x_{i}^{t}=0$, then the above constraint is enforced due to constraint (2.2). Constraints (2.5) can be aggregated in a similar fashion:

$$
\sum_{\tau=t+2}^{T} x_{j}^{\tau} \leq 1-x_{i}^{t}, \quad \forall(i, j) \in E, \forall t \in\{0, \cdots, T-2\},
$$

by observing that if a vertex $i$ is visited at time $t$, then its neighbors cannot be visited at any time later than $t+1$. On the other hand, if $x_{i}^{t}=0$, then the constraint is valid due to constraints (2.3).

The exact iterative algorithm of [13] solves a sequence of smaller IPs to obtain an optimal solution for the original problem. To ensure that formulations IP3 and IP3c are valid, we must set $T=|V|-1$, unless a better upper bound for the longest induced path is known. If the length $L^{*}$ of the induced path returned for some value of $T$ is strictly smaller than $T$, then $L^{*}$ is the optimal value for the original problem and the corresponding induced path is the longest one. Since there always exists an induced path whose length is equal to $\operatorname{diam}(G)$, the iterative search for the longest induced path may start from $T=\operatorname{diam}(G)+1$. If $L^{*} \ll|V|$, then each IP is expected to be solved much faster than the original formulation with the full upper bound. 
Matsypura et al. [13] also developed a randomized heuristic based on random walks. Its key idea consists in generating random walks that are also induced paths starting from each vertex $s \in S \subseteq V$, where $S$ is some predefined subset of vertices. The incumbent is initialized with an empty path. For each vertex $s \in S$ the heuristic builds $k$ random walks from $s$, where $k$ is a parameter. The longest among the $k$ random walks is returned, the incumbent is updated, and a new vertex $s \in S$ is explored. At the end, after all vertices in $S$ have been visited, the incumbent contain the longest path found by the heuristic. We observe that the heuristic is not efficient for small instances because, as the parameter $k$ grows, many repeated paths are generated starting at the same vertex.

Bökler et al. [5] proposed new integer linear programming formulations for the longest induced path problem, based on cut or subtour elimination constraints, exact separation routines, relaxation of variables and clique constraints. They obtain strictly stronger relaxations than those proposed in [13]. The core of their formulations is based on an extended graph $G^{*}=\left(V^{*}, E^{*}\right)$ by adding to $G$ a new (universal) vertex $s$ adjacent to all vertices of $V$, with $V^{*}=V \cup\{s\}$ and $E^{*}=E \cup\{(s, v): v \in V\}$. It seeks for a longest induced cycle through $s$ in $G^{*}$, ignoring induced chords incident to $s$. Searching for a cycle instead of a path, allows to require that each edge in the solution has exactly two adjacent edges that are also selected. Let $\delta^{*}(e) \subset E^{*}$ denote the edges adjacent to edge $e$ in $G^{*}$. The partial formulation ILP Base $_{\text {makes }}$ use of the binary variable $y_{e}$ associated to each edge $e \in E^{*}$ of the graph:

$$
\begin{aligned}
& y_{e}=y_{i, j}= \begin{cases}1, & \text { if and only if edge } e=(i, j) \text { is selected } \\
0, & \text { otherwise }\end{cases} \\
& \mathrm{ILP}_{\text {Base }}: \quad \max \sum_{e \in E} y_{e} \\
& \text { subject to: } \\
& \sum_{v \in V} y_{s, v}=2, \\
& 2 y_{e} \leq \sum_{f \in \delta^{*}(e)} y_{f} \leq 2, \\
& \forall e \in E, \\
& y_{e} \in\{0,1\} \text {, } \\
& \forall e \in E^{*} .
\end{aligned}
$$

The objective function (2.10) maximizes the number of edges in the resulting path. Constraint (2.11) enforces that exactly two edges incident to vertex $s$ are selected. To prevent the existence of chords, constraints (2.12) guarantee that any original edge $e \in E$ is adjacent to at most two selected edges. Furthermore, if edge $e \in E$ is selected, then precisely two of its adjacent edges must to be selected as well. Finally, constraints $(2.13)$ correspond to the integrality requirements.

However, this formulation is not enough, because it still allows solutions formed by multiple disjoint cycles, only one of them containing vertex $s$. In order to obtain a longest single cycle through $s$ yielding the longest induced path, one has to enforce that the graph induced by the optimal $y$-variables be connected. Bökler et al. [5] explored two strategies to achieve connectivity, by augmenting $\operatorname{ILP}_{\text {Base }}$ with cut or (generalized) subtour elimination constraints. Let $\delta^{*}(W)=\left\{(w, \bar{w}) \in E^{*}: w \in W, \bar{w} \in V^{*} \backslash W\right\}$ be the set of edges in the cut induced by $W \subseteq V^{*}$. The cut constraints can be formulated as:

$$
\sum_{e \in \delta^{*}(v)} y_{e} \leq \sum_{e \in \delta^{*}(W)} y_{e} \quad \forall W \subseteq V, \forall v \in W .
$$

They guarantee that if a vertex $v$ is incident to a selected edge, then any cut separating $v$ from $s$ contains at least two selected edges. Thus, there are (at least) two edge-disjoint paths selected between $v$ and $s$. Considering the cycle properties of ILP $\mathrm{I}_{\text {Base }}$, we may conclude that all selected edges form a common cycle through $s$. The enhanced formulation obtained by adding the cut constraints is named $\operatorname{ILP}_{\mathrm{Cut}}$. 
The above formulations can be further strengthened by introducing a set of additional clique inequalities. This, because if the subgraph $G[Q]=(Q, E[Q])$ induced in $G$ by some vertex subset $Q \subseteq V$ is a clique, then any induced path may contain at most one edge of $E[Q]$ :

$$
\sum_{e \in E[Q]} y_{e} \leq 1, \quad \forall Q \subseteq V: G[Q] \text { is a clique. }
$$

\section{ExaCt enumerative Algorithm AND A NEW HeURistic}

In this section, we propose an exact algorithm and a new heuristic, both of them based on the enumeration of all induced paths of the graph, exploring an approach similar to that in [13].

\subsection{Exact enumerative algorithm}

Algorithm 1 explores all vertices of the graph $G=(V, E)$ to be considered as the source vertex $s$ of an induced path. A recursive algorithm with backtracking is used to generate and explore all induced paths emanating from $s$. Procedure EXACT-ENUM takes as input the graph $G$. In lines 1 and 2 , the longest induced path and the current path are initialized, respectively. The loop in lines 3-5 explores all vertices of the graph. For each vertex $s \in V$, line 4 makes a call to the recursive procedure INDUCED-PATHS-FROM-VERTEX outlined in Algorithm 2 to compute all induced paths in $G$ emanating from the source $s$. Finally, line 6 returns the first longest induced path found during the exhaustive search.
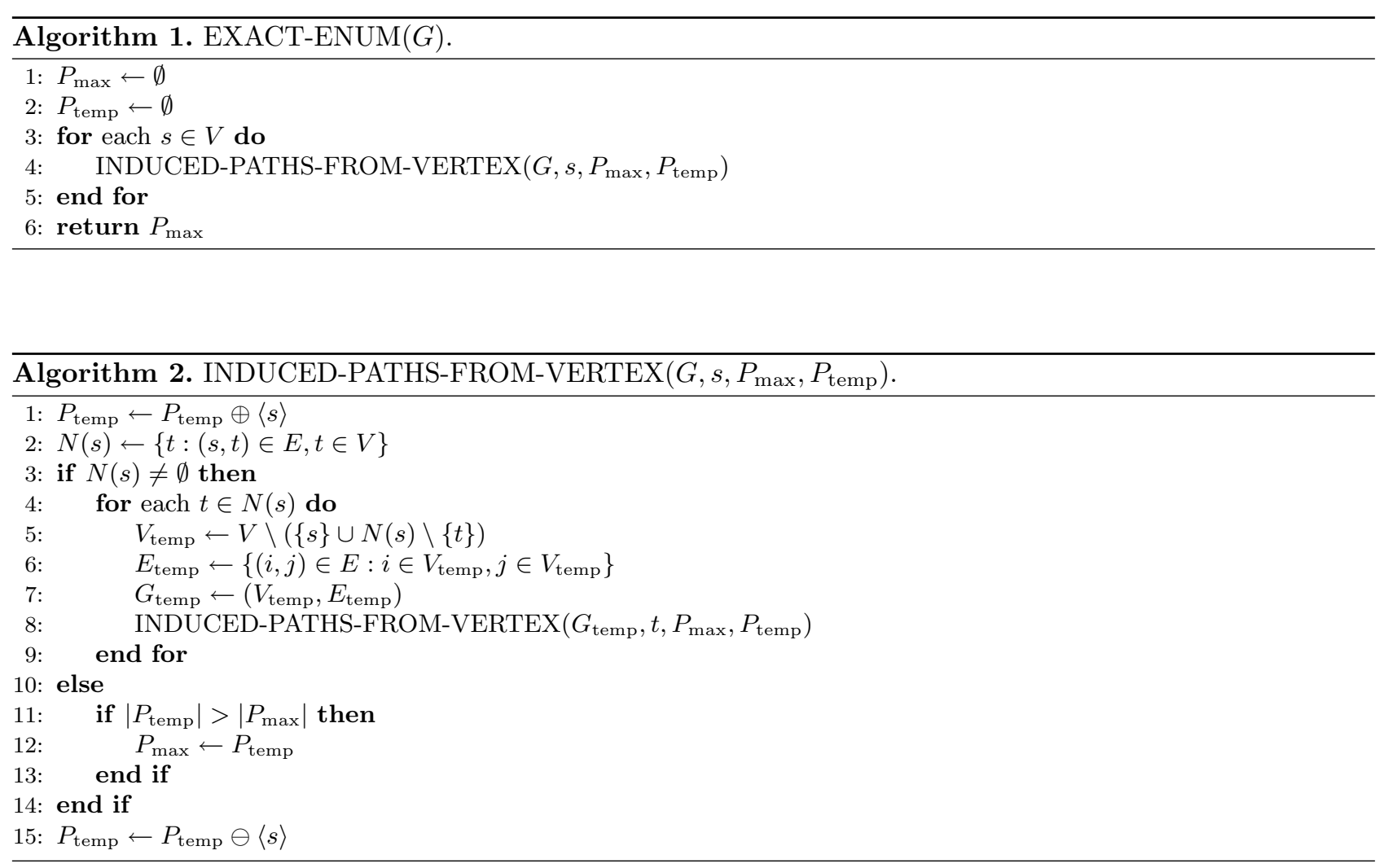

Procedure INDUCED-PATHS-FROM-VERTEX takes as inputs the graph $G$, the source vertex $s$, the longest

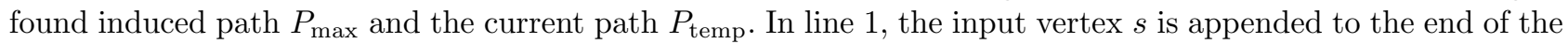


current path $P_{\text {temp }}$ under construction. Next, we obtain in line 2 the set of vertices $N(s)$ adjacent to vertex $s$. If $N(s)$ is not empty, then the loop in lines 4-9 visits every vertex $t$ adjacent to $s$. For each vertex $t$, lines $5-7$ create the subgraph $G_{\text {temp }}=\left(V_{\text {temp }}, E_{\text {temp }}\right)$ induced in $G$ by $V \backslash(\{s\} \cup N(s) \backslash\{t\})$, since the vertices in $N(s) \backslash\{t\}$ can not belong to any induced path containing $s$ and $t$. Vertex eliminations can be considered as cuts in the search space, because they discard the examination of paths that can not be induced paths. Finally, line 8 invokes recursively the procedure INDUCED-PATHS-FROM-VERTEX in the new induced graph $G_{\text {temp }}$ from the new source vertex $t$ that will be appended to the new, current path $P_{\text {temp }}$ obtained in line 1 . Otherwise, in case vertex $s$ has no neighbors, then it is a leaf of the search tree and the current path $P_{\text {temp }}$ can not be further extended. Then, line 11 checks if the size of path $P_{\text {temp }}$ is greater than that of path $P_{\max }$. If this is true, then we update $P_{\max }$ in line 12. At the end of procedure INDUCED-PATHS-FROM-VERTEX, in line 15, we remove from path $P_{\text {temp }}$ the vertex $s$ previously added in line 1 .

\subsection{Heuristic}

The new heuristic also explores all vertices of the graph $G=(V, E)$ as possible source vertices of induced paths. Procedure HLIPP whose pseudo-code appears in Algorithm 3 is very similar to Algorithm 1. However, it also takes as input an additional parameter maxpaths that limits the number of induced paths that are explored from each source vertex $s$. In lines 1 and 2 , the longest induced path and the current path are initialized, respectively. The loop in lines 3-8 explores all vertices of the graph. Variable \#paths is reset to 0 in line 4 at each iteration of the loop to count the number of induced paths explored from each source vertex $s \in V$. Variable last_improv is reset to 0 in line 5 before vertex $s$ is explored. Variable truncated is set to False in line 6 . Line 7 performs the call to the recursive procedure FIRST-INDUCED-PATHS. Line 9 returns the longest induced path $P_{\max }$ found.

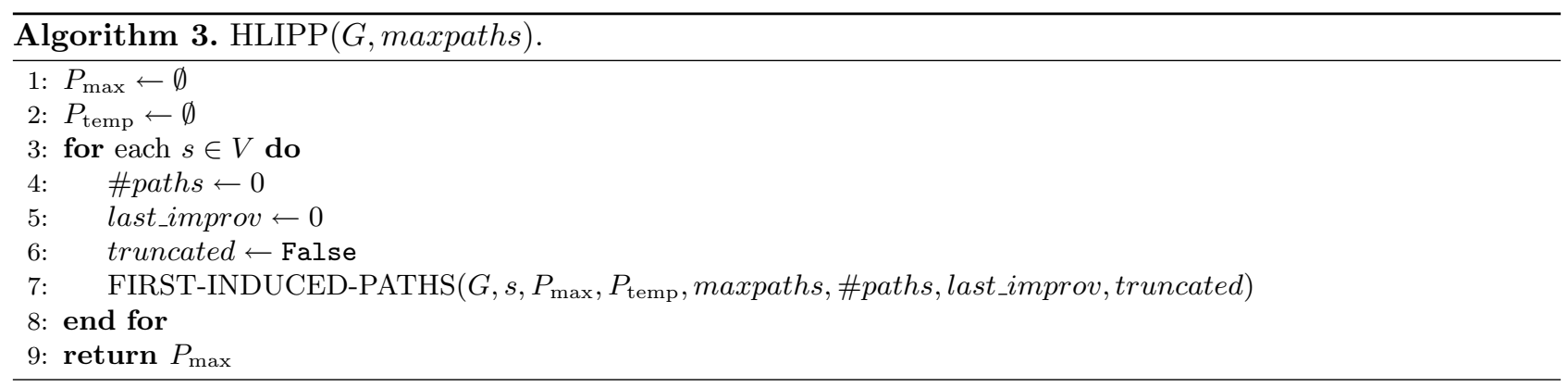

Procedure FIRST-INDUCED-PATHS outlined in Algorithm 4 takes as inputs the graph $G$, the source vertex $s$, the longest found induced path $P_{\max }$, the current path $P_{\text {temp }}$, the parameter maxpaths that is used as the stopping criterion to interrupt the search of the current vertex $s$, the counter \#paths of maximal induced paths generated from vertex $s$, the parameter last_improv that keeps track of the last time an improving solution was found, and a flag truncated that is used to indicate that the stopping criterion was reached for the vertex being explored. In line 1 we append the input vertex $s$ to the end of the current path $P_{\text {temp }}$ under construction. Next, we obtain in line 2 the set of vertices $N(s)$ adjacent to vertex $s$. If $N(s)$ is not empty, then the loop in lines 4-12 visits every vertex $t$ adjacent to $s$, unless a stopping criterion is met. For each vertex $t$, lines $5-7$ create de subgraph $G_{\text {temp }}=\left(V_{\text {temp }}, E_{\text {temp }}\right)$ induced in $G$ by $V \backslash(\{s\} \cup N(s) \backslash\{t\})$, since the vertices in $N(s) \backslash\{t\}$ can not belong to any induced path containing $s$ and $t$. Next, line 8 invokes recursively the procedure FIRSTINDUCED-PATHS in the new induced graph $G_{\text {temp }}$, from the new source vertex $t$ that will be appended to the new, current path $P_{\text {temp }}$ obtained in line 1 . If the stopping criterion for the exploration of vertex $s$ is reached during this call, then the value of the flag truncated is returned as True and propagated backwards in lines 9-11. Otherwise, in case vertex $s$ has no neighbors, then it is a leaf of the search tree and the current path $P_{\text {temp }}$ can 
not be further extended. Then, line 14 increases by one the number of maximal induced path generated. Next, line 15 checks if the size of path $P_{\text {temp }}$ is greater than the size of path $P_{\max }$. If this is true, then $P_{\max }$ is updated in line 16 and the variable last_improv, which accounts for the last time an improving solution was found, is updated in line 17. Line 19 implements the stopping criterion for the vertex under exploration. If it determines that more than maxpaths paths have been generated since the last improvement of the incumbent, then the search will be truncated in line 22, after emptying out $P_{\text {temp }}$ in line 20 and resetting the flag truncated to True in line 21. Finally, in line 25 at the end of procedure, vertex $s$ previously added in line 1 is removed from path $P_{\text {temp. }}$.

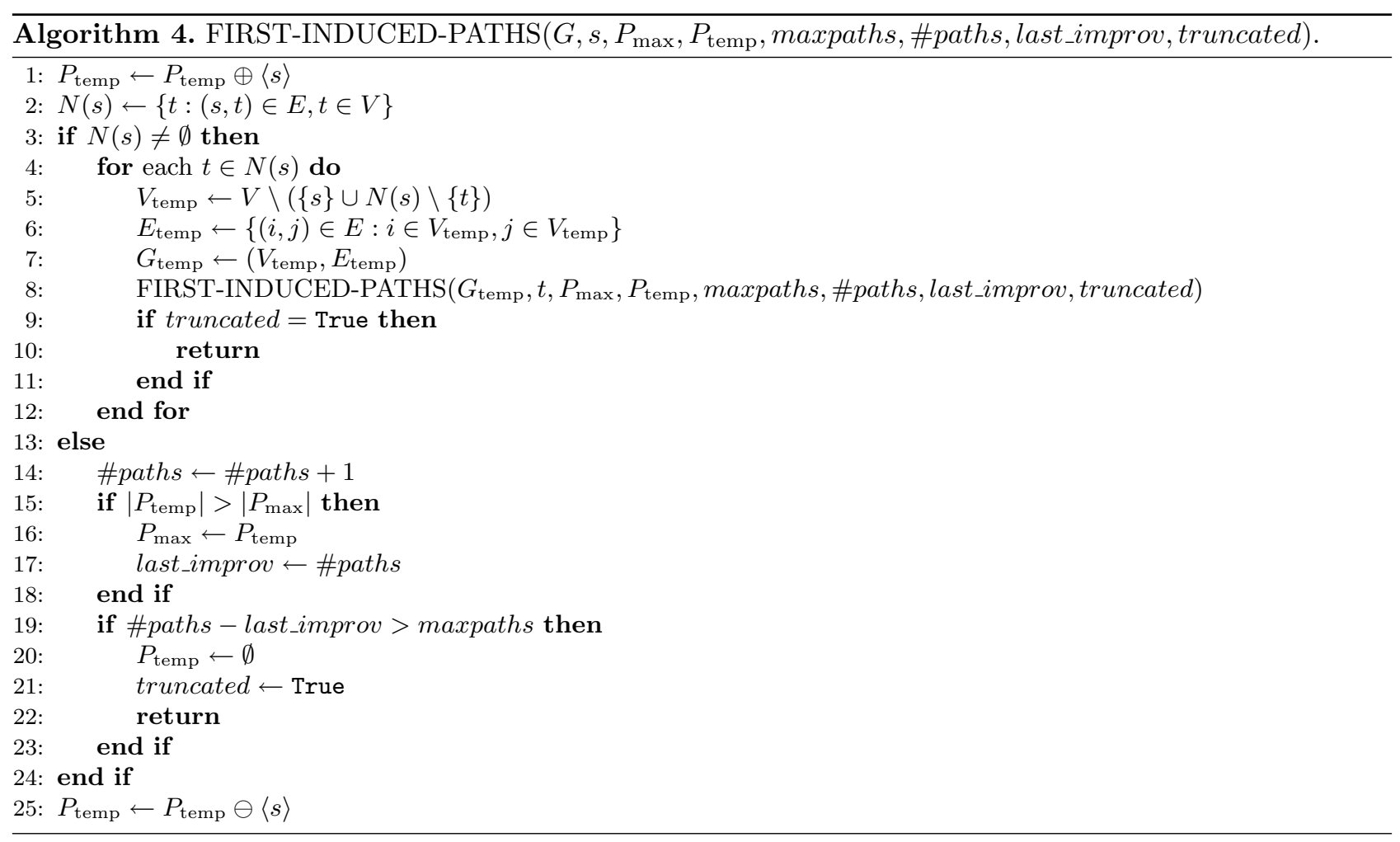

\section{Computational experiments}

In this section, we present computational experiments with the exact algorithm and the new heuristic proposed in Section 3 and the algorithms of Matsypura et al. [13] and Bökler et al. [5].

\subsection{Test instances}

Matsypura et al. [13] considered two sets of real-life graphs used in their computational experiments. The first set consists of network instances that are commonly used in the literature. They sought the longest induced path in the largest connected component of each instance. The second set is formed by networks representing the social connectivity of character interactions in different films and series [14]. They also generated synthetic instances with 20,30 and 40 vertices and different edge densities, using the Barabsi-Albert graph generating model [3].

Bökler et al. [5] considered new instances, in addition to those proposed by Matsypura et al. [13]. Their instances are grouped into four sets: RWC, MG, BAS and BAL. The first set (RWC) is a collection of 22 
TABLE 1. CPU performance comparison, data extracted from [17]: Higher values represent better performance. Second to last columns present the hardware used in this paper, in [13], and in [5], respectively.

\begin{tabular}{llll}
\hline \hline \multicolumn{1}{c}{ Benchmarks } & $\begin{array}{c}\text { Intel Core } \\
\text { i5-4460S }\end{array}$ & $\begin{array}{l}\text { Intel Xeon } \\
\text { E5-1650 v2 }\end{array}$ & $\begin{array}{l}\text { Intel Xeon } \\
\text { Gold 6134 }\end{array}$ \\
\hline Clock speed (GHz) & 2.9 & 3.5 & 3.2 \\
Turbo speed (GHz) & Up to 3.4 & Up to 3.9 & Up to 3.7 \\
CPU single thread rating & 1822 & 1929 & 2247 \\
CPU mark rating & 4383 & 8977 & 16687 \\
\hline
\end{tabular}

real-world networks, including communication and social networks of companies and of characters in books, as well as transportation, biological, and technical networks. The second set (MG) consists of 773 graphs representing social networks of movie characters, of which Matsypura et al. [13] considered only 17 of them. The instances in the other two sets were generated with the Barabsi-Albert probabilistic model for scale-free networks. The third set (BAS) has four groups of 30 graphs created with the same parameter values considered by Matsypura et al. [13], each group with $(|V|, d) \in\{(20,3),(30,3),(40,3),(40,2)\}$, where $d=(|E|+1) /|V|$ as defined by the authors. They also considered a fourth set (BAL) of graphs with 100 vertices and for each value of $d \in\{2,3,10,30,50\}$ they generated 30 instances.

\subsection{Computational environment}

We implemented the exact enumerative algorithm and the new heuristic proposed in Section 3 in $\mathrm{C}++$ with the GNU GCC compiler version 5.4.0. All experiments on these algorithms were performed on a computer with a four-processor $2.90 \mathrm{GHz}$ Intel Core i5-4460S CPU with $8 \mathrm{~GB}$ of RAM running the operating system Linux Ubuntu 16.04 LTS of 64 bits.

All experiments executed in [13] were conducted on a MacPro (late 2013) with a 3.5 GHz 6-Core Intel Xeon E5 and 32 GB of RAM running macOS the operating system High Sierra using a single thread. Their models were implemented in Anaconda Python 2.7 and Gurobi 8.1 was used as the IP solver. All Gurobi parameters were set to their default values, with each run limited to one single thread and a time limit of eight hours.

Bökler et al. [5] run all their computational tests on an Intel Xeon Gold 6134 machine with $3.2 \mathrm{GHz}$ and 256 GB of RAM running the operating system Debian 9. The authors used C++ (GCC 8.3.0) and limited each run to one single thread with a time limit of 20 min and a memory limit of 8 GB of RAM.

Table 1 based on the benchmarks in [17] addresses the performance of the computers involved in the three experiments. Although the computers used by Matsypura et al. [13] and Bökler et al. [5] are faster than ours, we will compare the execution times of the algorithms on their own, independently of the machines on where the experiments had been executed. In other words, we will not take into account the differences in performance of the three different processors. For a more detailed evaluation, the interested reader may use the information in this table.

\subsection{Exact algorithms}

In this section, we compare our exact enumerative algorithm with the state-of-the-art integer programming approaches proposed by Matsypura et al. [13] and Bökler et al. [5], with a time limit of $20 \mathrm{~min}$ and one single thread for each run.

Bökler et al. [5] denoted by "W" their implementation of the exact algorithm in [13]. The authors considered various parameter settings in their implementation of model $\mathrm{ILP}_{\mathrm{Cut}}$, with all resulting algorithms denoted by a "C" with subscripts and superscripts defining the parameters: subscript "frac" denotes the use of fractional separation in addition to integral separation; superscript " $\mathrm{n}$ " shows that vertex variables are the unique integer 
TABLE 2. Running times in seconds of the exact algorithms on the RWC instances.

\begin{tabular}{|c|c|c|c|c|c|c|c|c|c|}
\hline Instance & $\mathrm{OPT}$ & $|V|$ & $|E|$ & EXACT-ENUM & $W$ & Tmin & Cmin & Tmax & Cmax \\
\hline high-tech & 13 & 33 & 91 & 0.15 & 15.40 & 0.41 & $C_{\text {int }}^{\mathrm{n}, \mathrm{c}}$ & 3.15 & $C_{\text {frac }}^{\mathrm{c}}$ \\
\hline karate & 9 & 34 & 78 & 0.04 & 2.98 & 0.66 & $C_{\mathrm{int}}^{\mathrm{n}, \mathrm{c}}$ & 3.71 & $C_{\text {frac }}^{\mathrm{n}}$ \\
\hline mexican & 16 & 35 & 117 & 0.81 & 73.30 & 0.87 & $C_{\mathrm{int}}^{\mathrm{n}, \mathrm{c}}$ & 3.59 & $C_{\text {frac }}^{\mathrm{c}}$ \\
\hline sawmill & 18 & 36 & 62 & 0.06 & 70.00 & 0.43 & $C_{\text {frac }}^{\text {int }}$ & 3.34 & $C_{\text {frac }}^{\text {nrac }}$ \\
\hline tailorS1 & 13 & 39 & 158 & 1.05 & 83.80 & 1.51 & $C_{\mathrm{int}}^{\mathrm{n}}$ & 7.92 & $C_{\text {frac }}$ \\
\hline chesapeake & 16 & 39 & 170 & 0.78 & 106.00 & 1.84 & $C_{\text {int }}$ & 13.11 & $C_{\text {frac }}$ \\
\hline tailors2 & 15 & 39 & 223 & 2.13 & 445.00 & 2.89 & $C_{\mathrm{int}}^{\mathrm{n}, \mathrm{c}}$ & 21.78 & $C_{\text {frac }}$ \\
\hline attiro & 31 & 59 & 128 & 71.35 & () & 0.89 & $C_{\mathrm{int}}^{\mathrm{n}, \mathrm{c}}$ & 2.57 & $C_{\text {frac }}$ \\
\hline krebs & 17 & 62 & 153 & 0.80 & 522.00 & 2.33 & $C_{\text {frac }}^{\mathrm{n}, \mathrm{c}}$ & 28.21 & $C_{\text {frac }}$ \\
\hline dolphins & 24 & 62 & 159 & 91.77 & (ㄷ) & 2.99 & $\begin{array}{l}\text { frac } \\
C_{\text {frac }}^{\mathrm{n}}\end{array}$ & 27.59 & $C_{\text {frac }}$ \\
\hline prison & 36 & 67 & 142 & 64.15 & (๖) & 1.02 & $C_{\mathrm{int}}^{\mathrm{n}, \mathrm{c}}$ & 13.36 & $C_{\text {int }}$ \\
\hline huck & 9 & 69 & 297 & 0.30 & 41.70 & 5.96 & $C_{\mathrm{int}}^{\mathrm{n}, \mathrm{c}}$ & () & $C_{\text {int }}$ \\
\hline sanjuansur & 38 & 75 & 144 & 645.81 & (๖) & 3.65 & $C_{\text {frac }}^{\mathrm{n}}$ & 30.67 & $C_{\text {int }}$ \\
\hline jean & 11 & 77 & 254 & 1.18 & 121.00 & 3.88 & $C_{\mathrm{int}}^{\mathrm{n}, \mathrm{c}}$ & 464.89 & $C_{\text {int }}$ \\
\hline david & 19 & 87 & 406 & 23.35 & () & 6.93 & $C_{\mathrm{int}}^{\mathrm{n}, \mathrm{c}}$ & 719.46 & $C_{\text {frac }}$ \\
\hline ieeebus & 47 & 118 & 179 & 322.40 & (๑) & 3.13 & $C_{\text {frac }}^{\mathrm{n}}$ & 39.82 & $C_{\mathrm{int}}^{\mathrm{c}}$ \\
\hline sfi & 13 & 118 & 200 & 0.32 & 44.40 & 2.44 & $C_{\text {frac }}^{\mathrm{n}, \mathrm{c}}$ & 47.41 & $C_{\text {int }}$ \\
\hline anna & 20 & 138 & 493 & 17.17 & () & 7.09 & $C_{\mathrm{int}}^{\mathrm{n}, \mathrm{c}}$ & 439.23 & $C_{\mathrm{int}}^{\mathrm{n}}$ \\
\hline usair & 46 & 332 & 2126 & (๖) & (b) & 922.94 & $C_{\text {int }}^{\text {nint }}$ & (ㄷ) & all but $C_{\text {int }}^{\mathrm{n}, \mathrm{c}}$ \\
\hline 494bus & 142 & 494 & 586 & (ค) & (๖) & 170.74 & $C_{\text {frac }}^{\mathrm{n}, \mathrm{c}}$ & () & $\mathrm{C}_{\text {int }}, \mathrm{C}_{\mathrm{int}}^{\mathrm{c}}, \mathrm{C}_{\mathrm{int}}^{\mathrm{n}}, \mathrm{C}_{\mathrm{int}}^{\mathrm{n}, \mathrm{c}}$ \\
\hline
\end{tabular}

variables. The superscript "c" indicates the use of clique constraints. The authors considered thereby all possible combinations of parameters of ILP $_{\text {Cut }}$, corresponding to eight different implementations.

Table 2 displays the computational experiments on the RWC instances (as named by Bökler et al. [5]). The second column gives the optimal value. The third and fourth columns show the number of vertices and edges in each instance, respectively. The fifth column displays the time in seconds taken by our exact enumerative algorithm. The sixth column shows the time in seconds obtained by the exact algorithm of [13], as implemented in [5]. The next two columns, indicated respectively by Tmin and Cmin, present the minimum running time over all eight implementations of ILP ${ }_{\mathrm{Cut}}$ and the fastest variant. The last two columns, indicated respectively by Tmax and Cmax, present the maximum running time over all eight implementations of ILP $_{\text {Cut }}$ and the slowest variant (or those that timed out). Timeouts are denoted by $\odot$ ) and the minimum times were marked in boldface. Instances yeast and 622bus (not presented in the table) could not be solved by any of the algorithms within the maximum time limit.

The table shows that, for the RWC instances, the new enumerative algorithm performed better in terms of running times and the number of instances solved to optimality than the exact algorithm in [13]. However, the comparison with respect to the implementations of $\mathrm{ILP}_{\mathrm{Cut}}$ is less straightforward. It is noticeable to observe that the fastest and the slowest variants of $\operatorname{ILP}_{\mathrm{Cut}}$ change from instance to instance. Variant $C_{\text {int }}^{n, c}$ was the

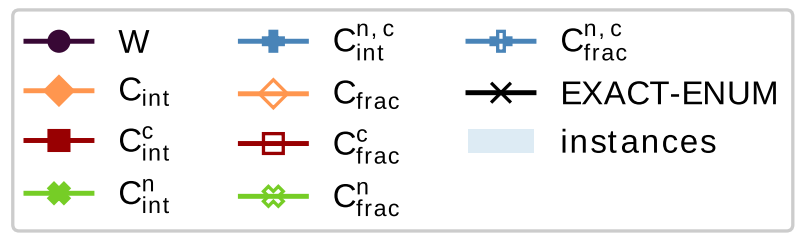

FIgURE 1. Legend with the identifications of the algorithms and ILP $_{\text {Cut }}$ implementations. 


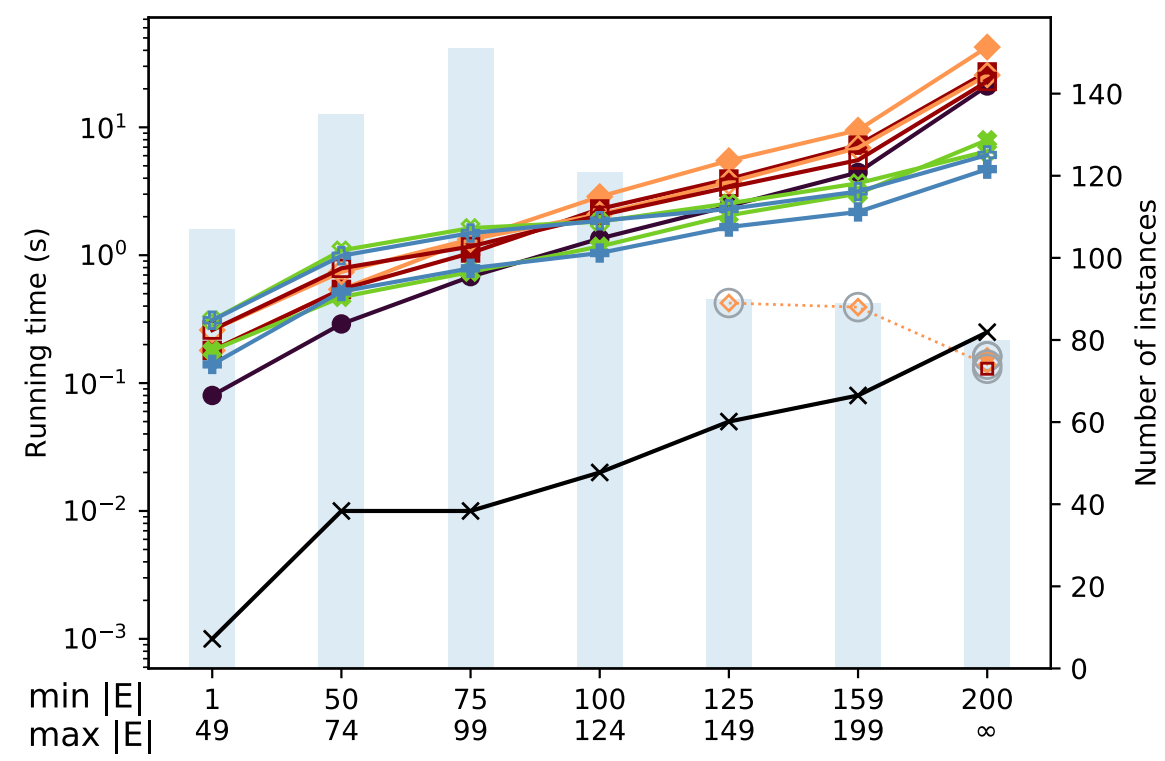

FiguRE 2. Running times in seconds of the exact algorithms and the ILP implementations on the MG instances.

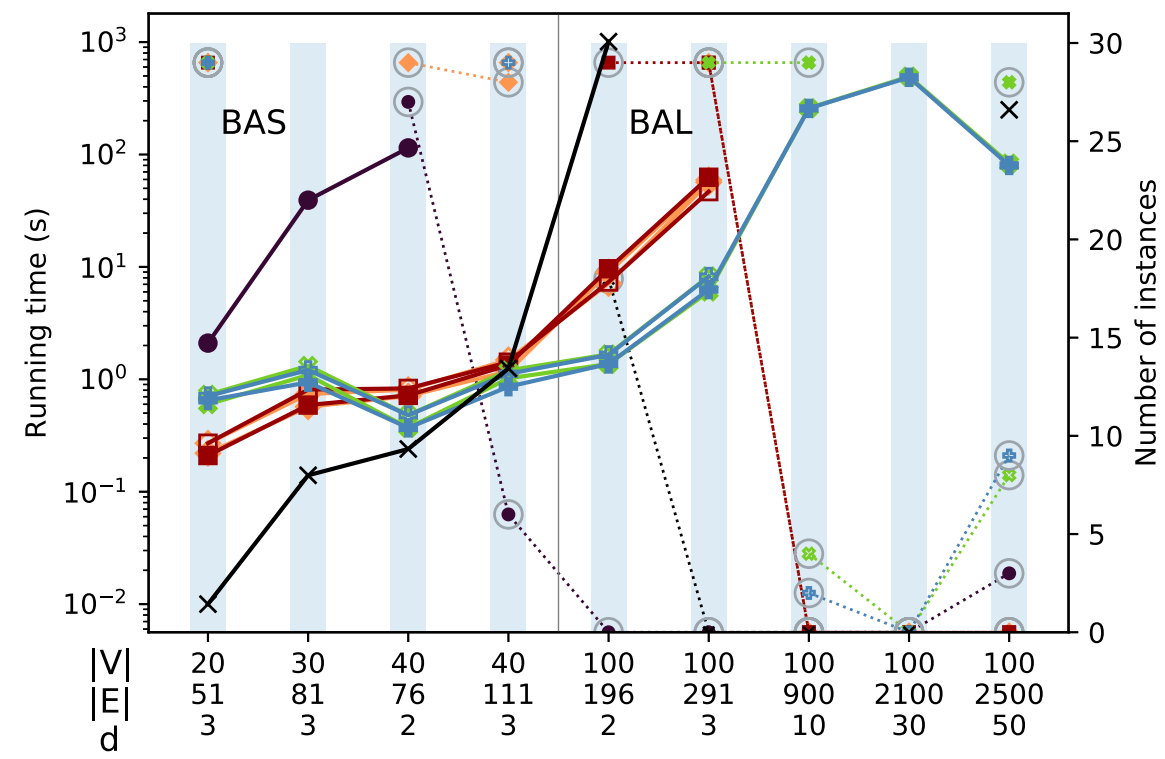

FiguRE 3. Running times in seconds of the exact algorithms and the ILP implementations on the BAS and BAL instances. 


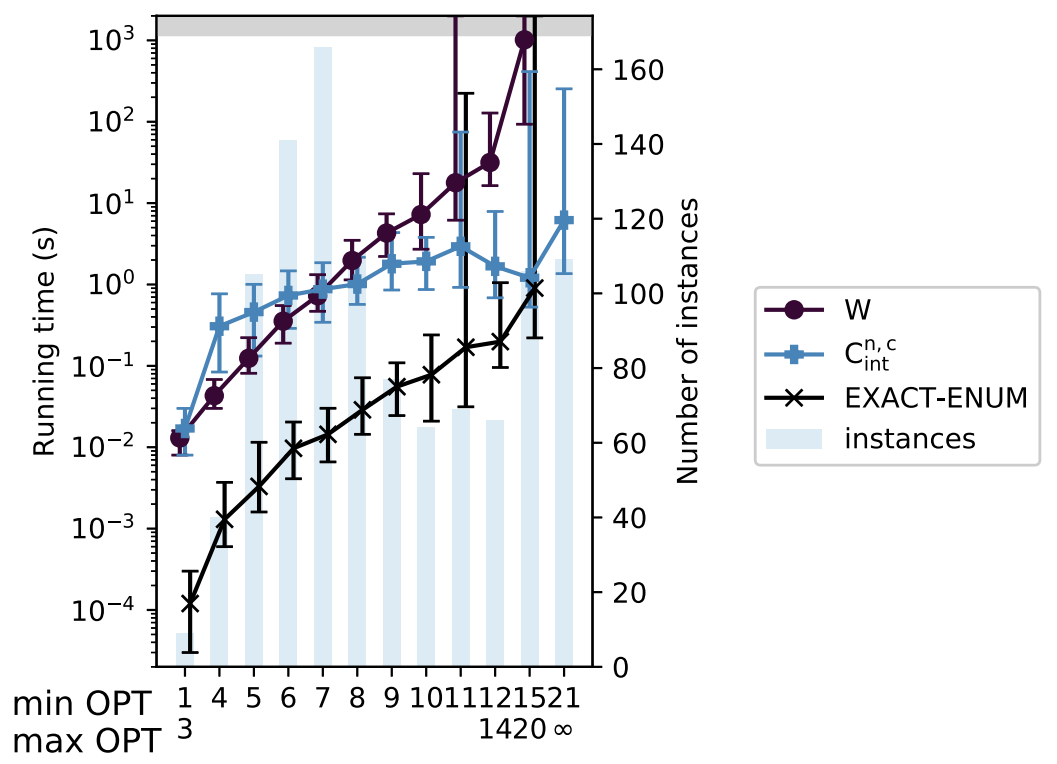

FIGURE 4. Running times vs. optimal values for all instances: whiskers mark the $20 \%$ and $80 \%$ percentiles. The gray area on top of the plot marks timeouts. Each run was limited to 20 min.

fastest for 11 out of 22 instances and the only to solve usair, but did not solve instance 494bus within the time limit. Variants $C_{\mathrm{int}}^{c}$ and $C_{\mathrm{frac}}^{c}$ never were the fastest. The new enumerative algorithm was faster than the fastest variant of ILP $_{\mathrm{Cut}}$ for 11 out of 22 instances. For two instances, the new algorithm was slower than the fastest, but faster than the slowest variant of ILP $_{\text {Cut }}$. The new enumerative algorithm was slower than the worst variant of ILP $_{\text {Cut }}$ for five out of the 22 RWC instances. Still, for two instances the new enumerative algorithm did not find the optimal solution, but the fastest variant of $\operatorname{ILP}_{\mathrm{Cut}}$ did. Overall, we may say that the new enumerative algorithm is competitive with the integer programming formulations ILP $_{\mathrm{Cut}}$ in [5], in particular if one considers the differences in performance of the processors involved in the computational experiments and the fact that the best variant of $\operatorname{ILP}_{\mathrm{Cut}}$ for each instance can not be predicted beforehand and choosing the best among the eight ILP $_{\text {Cut }}$ implementations is way far from being a clear decision.

Figure 1 shows the legend with the identification of the algorithms and the ILP $_{\text {Cut }}$ implementations whose results are displayed in Figures 2 and 3. Figure 2 displays comparative results for the MG instances, with the horizontal axis indicating the minimum and the maximum number of edges in the instances in each category (same abscissa). Figure 3 shows the results for the instances in sets BAS and BAL, with the horizontal axis indicating the number of vertices, the number of edges, and the parameter $d$ for the instances in each category (same abscissa). Vertical bars in light blue in the background give the number of instances in each category. For each exact algorithm ( $W$, the eight variants of $\mathrm{ILP}_{\mathrm{Cut}}$, and the new enumerative algorithm), we represent the median of the running times over all instances in the same category. Points represented by gray encircled markers connected by dotted lines show the number of solved instances (when not all in the same category have been solved to optimality).

We observe in Figure 2 that the new exact enumerative algorithm solved all 773 MG instances using the smallest running times, with respect to all other algorithms. Figure 3 shows that the new exact enumerative algorithm remains competitive and globally the fastest on average also for the BAS instances. However, both the enumerative algorithm and that in [13] do not perform well for the BAL instances, where the latter failed for virtually all instances, while some implementations of ILP $_{\mathrm{Cut}}$ were faster and solved more instances than the new exact enumerative algorithm. 
TABLE 3. Comparative numerical results between the randomized heuristic [13] and heuristic HLIPP on large graphs.

\begin{tabular}{|c|c|c|c|c|c|c|c|c|c|c|c|c|c|c|c|}
\hline \multirow{3}{*}{ Name } & \multirow{3}{*}{$|V|$} & \multirow{3}{*}{$|E|$} & \multirow{3}{*}{ OPT } & \multicolumn{6}{|c|}{ Randomized heuristic } & \multicolumn{6}{|c|}{ HLIPP } \\
\hline & & & & \multicolumn{2}{|c|}{$\mathrm{H} 100$} & \multicolumn{2}{|c|}{$\mathrm{H} 1000$} & \multicolumn{2}{|c|}{ H10000 } & \multicolumn{2}{|c|}{ LH100 } & \multicolumn{2}{|c|}{ LH1000 } & \multicolumn{2}{|c|}{ LH10000 } \\
\hline & & & & Time (s) & Obj. & Time (s) & Obj. & Time (s) & Obj. & Time (s) & Obj. & Time (s) & Obj. & Time (s) & Obj. \\
\hline abyss & 28 & 108 & 6 & 0.21 & 6 & 2.1 & 6 & 21 & 6 & 0.01 & 6 & 0.01 & 6 & 0.01 & 6 \\
\hline hi-tech & 33 & 91 & 13 & 0.39 & 13 & 3.8 & 13 & 37 & 13 & 0.03 & 13 & 0.15 & 13 & 0.15 & 13 \\
\hline karate & 34 & 78 & 9 & 0.31 & 9 & 2.9 & 9 & 30 & 9 & 0.02 & 9 & 0.04 & 9 & 0.04 & 9 \\
\hline mexican & 35 & 117 & 16 & 0.53 & 15 & 5.3 & 15 & 53 & 16 & 0.04 & 16 & 0.36 & 16 & 0.76 & 16 \\
\hline sawmill & 36 & 62 & 18 & 0.41 & 18 & 4.1 & 18 & 41 & 18 & 0.04 & 18 & 0.06 & 18 & 0.05 & 18 \\
\hline pulp fiction & 38 & 102 & 7 & 0.27 & 7 & 2.6 & 7 & 26 & 7 & 0.02 & 7 & 0.02 & 7 & 0.02 & 7 \\
\hline chesapeake & 39 & 170 & 16 & 0.56 & 16 & 5.5 & 16 & 55 & 16 & 0.05 & 16 & 0.42 & 16 & 0.73 & 16 \\
\hline tailorS1 & 39 & 158 & 13 & 0.58 & 12 & 5.7 & 13 & 58 & 13 & 0.05 & 13 & 0.38 & 13 & 1.00 & 13 \\
\hline tailorS2 & 39 & 223 & 15 & 0.64 & 13 & 6.4 & 14 & 64 & 15 & 0.06 & 12 & 0.49 & 13 & 1.97 & 15 \\
\hline romeo and juliet & 41 & 120 & 9 & 0.35 & 8 & 3.5 & 9 & 35 & 9 & 0.03 & 9 & 0.07 & 9 & 0.06 & 9 \\
\hline oceans 12 & 42 & 147 & 8 & 0.40 & 8 & 3.9 & 8 & 39 & 8 & 0.04 & 8 & 0.07 & 8 & 0.07 & 8 \\
\hline die hard & 47 & 237 & 10 & 0.50 & 9 & 5.0 & 10 & 49 & 10 & 0.05 & 10 & 0.11 & 10 & 0.11 & 10 \\
\hline star wars II & 47 & 148 & 8 & 0.41 & 8 & 4.1 & 8 & 41 & 8 & 0.04 & 8 & 0.06 & 8 & 0.05 & 8 \\
\hline oceans 11 & 50 & 145 & 9 & 0.51 & 9 & 5.0 & 9 & 48 & 9 & 0.05 & 8 & 0.08 & 9 & 0.07 & 9 \\
\hline the departed & 51 & 117 & 8 & 0.45 & 8 & 4.5 & 8 & 45 & 8 & 0.04 & 8 & 0.05 & 8 & 0.05 & 8 \\
\hline krebs & 62 & 153 & 17 & 0.78 & 13 & 7.7 & 16 & 77 & 17 & 0.09 & 15 & 0.59 & 17 & 0.75 & 17 \\
\hline philadelphia & 65 & 258 & 10 & 0.68 & 8 & 6.8 & 9 & 67 & 9 & 0.09 & 9 & 0.31 & 10 & 0.30 & 10 \\
\hline 2012 & 66 & 211 & 12 & 0.86 & 10 & 8.4 & 11 & 84 & 12 & 0.09 & 11 & 0.62 & 12 & 0.70 & 12 \\
\hline braveheart & 67 & 310 & 11 & 0.82 & 9 & 8.1 & 9 & 81 & 10 & 0.09 & 11 & 0.60 & 11 & 0.64 & 11 \\
\hline huck & 69 & 297 & 9 & 0.75 & 8 & 7.4 & 9 & 74 & 9 & 0.11 & 9 & 0.30 & 9 & 0.28 & 9 \\
\hline gandhi & 76 & 200 & 10 & 0.89 & 9 & 8.8 & 10 & 88 & 10 & 0.11 & 9 & 0.16 & 10 & 0.15 & 10 \\
\hline watchmen & 76 & 201 & 9 & 0.79 & 9 & 7.8 & 9 & 80 & 9 & 0.10 & 9 & 0.26 & 9 & 0.24 & 9 \\
\hline jean & 77 & 254 & 11 & 0.97 & 10 & 9.6 & 11 & 96 & 11 & 0.12 & 11 & 0.80 & 11 & 1.10 & 11 \\
\hline godfather II & 78 & 219 & 18 & 1.03 & 13 & 10.2 & 14 & 103 & 15 & 0.12 & 16 & 0.90 & 18 & 1.23 & 18 \\
\hline catch me if you can & 82 & 162 & 8 & 0.94 & 8 & 9.3 & 8 & 93 & 8 & 0.11 & 8 & 0.17 & 8 & 0.15 & 8 \\
\hline david & 87 & 406 & 19 & 1.48 & 14 & 14.8 & 15 & 149 & 17 & 0.23 & 18 & 1.87 & 19 & 14.40 & 19 \\
\hline doors & 95 & 567 & 12 & 1.65 & 9 & 16.3 & 11 & 163 & 12 & 0.30 & 12 & 2.17 & 12 & 2.75 & 12 \\
\hline public enemies & 99 & 317 & 20 & 1.55 & 12 & 15.5 & 15 & 154 & 15 & 0.20 & 17 & 1.57 & 20 & 3.58 & 20 \\
\hline santafe & 118 & 200 & 13 & 1.39 & 9 & 13.9 & 10 & 138 & 10 & 0.19 & 13 & 0.30 & 13 & 0.29 & 13 \\
\hline anna & 138 & 493 & 20 & 3.02 & 13 & 30.0 & 15 & 303 & 16 & 0.42 & 16 & 3.36 & 20 & 16.01 & 20 \\
\hline attiro & 59 & 128 & 31 & 1.53 & 27 & 15 & 28 & 142 & 29 & 0.11 & 28 & 0.92 & 29 & 8.77 & 30 \\
\hline dolphins & 62 & 159 & 24 & 1.38 & 21 & 14 & 22 & 137 & 24 & 0.11 & 20 & 0.98 & 22 & 9.11 & 23 \\
\hline prison & 67 & 142 & 36 & 1.52 & 27 & 15 & 30 & 152 & 32 & 0.13 & 28 & 1.23 & 31 & 11.66 & 36 \\
\hline sanjuansur & 75 & 144 & 38 & 2.09 & 30 & 21 & 33 & 213 & 36 & 0.16 & 35 & 1.42 & 35 & 14.26 & 37 \\
\hline ieeebus & 118 & 179 & 47 & 3.29 & 29 & 33 & 31 & 329 & 38 & 0.37 & 44 & 3.20 & 44 & 30.95 & 47 \\
\hline USAir97 & 332 & 2126 & 46 & 21.07 & 22 & 206 & 27 & 2044 & 30 & 3.50 & 29 & 37.88 & 33 & 347.12 & 38 \\
\hline 494_bus & 494 & 586 & 142 & 41.56 & 45 & 413 & 49 & 3901 & 61 & 7.99 & 101 & 70.25 & 107 & 738.86 & 114 \\
\hline 662_bus & 662 & 906 & $?$ & 141.00 & 90 & 1402 & 92 & 13819 & 110 & 26.33 & 242 & 172.08 & 242 & 1608.83 & 242 \\
\hline S.Cerevisae & 1458 & 1948 & $?$ & 290.62 & 35 & 2855 & 38 & 28221 & 43 & 76.60 & 144 & 451.74 & 149 & 4139.20 & 155 \\
\hline \# of optimal values & & & 37 & & 12 & & 18 & & 24 & & 20 & & 29 & & 32 \\
\hline
\end{tabular}

Bökler et al. [5] observed that the running time of the exact algorithm in [13] heavily depends on the optimal value of each instance, while their new implementations $\left(e . g . C_{\text {int }}^{n, c}\right)$ are less dependent on the solution size. This is illustrated in Figure 4, which correlates the median running times of three exact algorithms with the size of the longest induced path (OPT), considering all the test instances solved by at least one of the exact methods. The horizontal axis of the figure indicates the minimum and the maximum optimal values for the instances represented in the same category with the same abscissa. The running time of the new exact enumerative algorithm is naturally expected to depend on the optimal value, as illustrated by the figure.

\subsection{Heuristics}

In this set of experiments, we compare the performance of the HLIPP heuristic described in Algorithm 3 with the randomized heuristic of Matsypura et al. [13] on the same instances considered by its authors. 
TABLE 4. Comparative numerical results between the exact algorithm EXACT-ENUM and the heuristic HLIPP (LH10000 corresponds to Algorithm 3 with 10000 as the stopping criterion).

\begin{tabular}{|c|c|c|c|c|}
\hline \multirow[b]{2}{*}{ Name } & \multicolumn{2}{|c|}{ EXACT-ENUM } & \multicolumn{2}{|c|}{ HLIPP (LH10000) } \\
\hline & OPT & Time (s) & Time $(\mathrm{s})$ & Obj. \\
\hline abyss & 6 & 0.01 & 0.01 & 6 \\
\hline hi-tech & 13 & 0.15 & 0.15 & 13 \\
\hline karate & 9 & 0.04 & 0.04 & 9 \\
\hline mexican & 16 & 0.81 & 0.76 & 16 \\
\hline sawmill & 18 & 0.06 & 0.05 & 18 \\
\hline pulp fiction & 7 & 0.02 & 0.02 & 7 \\
\hline chesapeake & 16 & 0.78 & 0.73 & 16 \\
\hline tailorS1 & 13 & 1.05 & 1.00 & 13 \\
\hline tailorS2 & 15 & 2.13 & 1.97 & 15 \\
\hline romeo and juliet & 9 & 0.06 & 0.06 & 9 \\
\hline oceans 12 & 8 & 0.07 & 0.07 & 8 \\
\hline die hard & 10 & 0.12 & 0.11 & 10 \\
\hline star wars II & 8 & 0.06 & 0.05 & 8 \\
\hline oceans 11 & 9 & 0.07 & 0.07 & 9 \\
\hline the departed & 8 & 0.05 & 0.05 & 8 \\
\hline krebs & 17 & 0.80 & 0.75 & 17 \\
\hline philadelphia & 10 & 0.31 & 0.30 & 10 \\
\hline 2012 & 12 & 0.71 & 0.70 & 12 \\
\hline braveheart & 11 & 0.66 & 0.64 & 11 \\
\hline huck & 9 & 0.30 & 0.28 & 9 \\
\hline gandhi & 10 & 0.16 & 0.15 & 10 \\
\hline watchmen & 9 & 0.25 & 0.24 & 9 \\
\hline jean & 11 & 1.18 & 1.10 & 11 \\
\hline godfather II & 18 & 1.26 & 1.23 & 18 \\
\hline catch me if you can & 8 & 0.17 & 0.15 & 8 \\
\hline david & 19 & 23.35 & 14.40 & 19 \\
\hline doors & 12 & 2.77 & 2.75 & 12 \\
\hline public enemies & 20 & 3.60 & 3.58 & 20 \\
\hline santafe & 13 & 0.32 & 0.29 & 13 \\
\hline anna & 20 & 17.17 & 16.01 & 20 \\
\hline attiro & 31 & 71.35 & 8.77 & 30 \\
\hline dolphins & 24 & 91.77 & 9.11 & 23 \\
\hline prison & 36 & 64.15 & 11.66 & 36 \\
\hline sanjuansur & 38 & 645.81 & 14.26 & 37 \\
\hline ieeebus & 47 & 322.40 & 30.95 & 47 \\
\hline USAir97 & 46 & (๑) & 347.12 & 38 \\
\hline 494_bus & 142 & () & 738.86 & 114 \\
\hline 662_bus & $?$ & () & 1608.83 & 242 \\
\hline S.Cerevisae & $?$ & () & 4139.20 & 155 \\
\hline \# of optimal values & 37 & & & 32 \\
\hline
\end{tabular}

Comparative computational results are presented in Table 3. The four first columns present the name of the instance, the number of vertices, the number of edges, and the size of the longest induced path (OPT) for each graph (unknown for the two last instances). Next, each pair of columns give the time in seconds and the best solution value obtained by one run of the randomized heuristic [13] with 100, 1000 and 10000 restarts (columns H100, H1000, and H10000, respectively). Columns LH100, LH1000 and LH10000 give the same information for 
Algorithm 3, with 100, 1000 and 10000 as the limit on the number of paths (maxpaths) that are explored from each source vertex, respectively.

The randomized heuristic stops after generating a given number of random induced paths (named "restarts" by the authors) starting from each vertex of some given subset $S \subseteq V$. Therefore, it implements diversification by randomization. The proposed HLIPP deterministic heuristic stops after generating a sequence of maxpaths induced paths from each vertex of the graph that do not improve the incumbent solution. Our approach explores more induced paths, leading to a potentially better solution. In addition, it generates the induced paths more efficiently, which makes HLIPP faster for all test instances in the computational experiments.

In fact, Table 3 shows that the running times of the proposed HLIPP heuristic are consistently smaller than those reported for the randomized heuristic in [13]. HLIPP is also more robust, since it found significantly more optimal values (or the best known solution values). The quality of heuristic HLIPP and the difference in performance of the two heuristics in terms of solution quality and running times are particularly clear for the two last instances with unknown optimal value in the bottom of the table.

Table 4 compares the exact algorithm EXACT-ENUM (Algorithm 1) with heuristic HLIPP (Algorithm 3) on all 39 instances in Table 3. Heuristic HLIPP (running with maxpaths $=10000$ ) found the optimal value for 32 instances (out of 37 whose optimal values are known) in time not greater than that taken by our exact algorithm.

\section{AnAlysis OF THE OPtimal SOLUtions}

In this section, we investigate the correlation of the running times and sizes of the longest induced paths with some characteristics of the solved instances of the four test sets (RWC, MG, BAS, BAL) described in Section 4.1. We also analyze the eccentricity and the degree of the extreme vertices of the optimal solutions obtained with our exact algorithm.

Figure 5 shows the Pearson correlation coefficient [18] between the running times of the instances solved by our exact enumeration algorithm and some graph characteristics: average vertex degree, average vertex eccentricity, average pairwise distance, global clustering coefficient, and average vertex (local) clustering coefficient. The global clustering coefficient is the ratio between three times the number of triangles and the number of pairs of adjacent edges in a graph [19]. This measure is also called the transitivity and represents the probability that two vertices that are adjacent to a third vertex will be adjacent themselves [16]. The (local) clustering coefficient

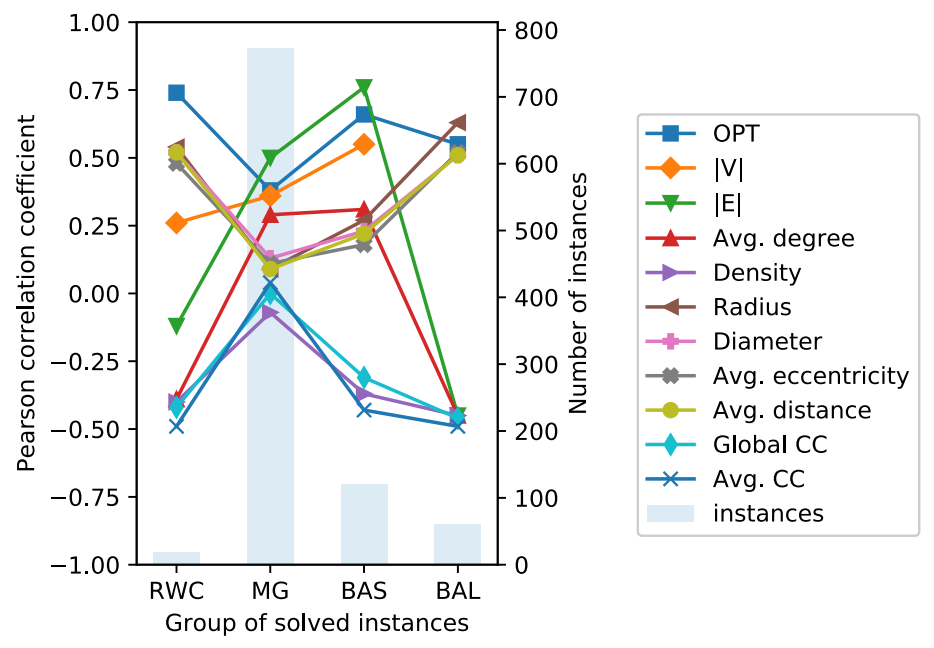

Figure 5. Pearson correlation coefficient [18] between the running times of the instances solved by our exact enumeration algorithm EXACT-ENUM and some graph characteristics. 


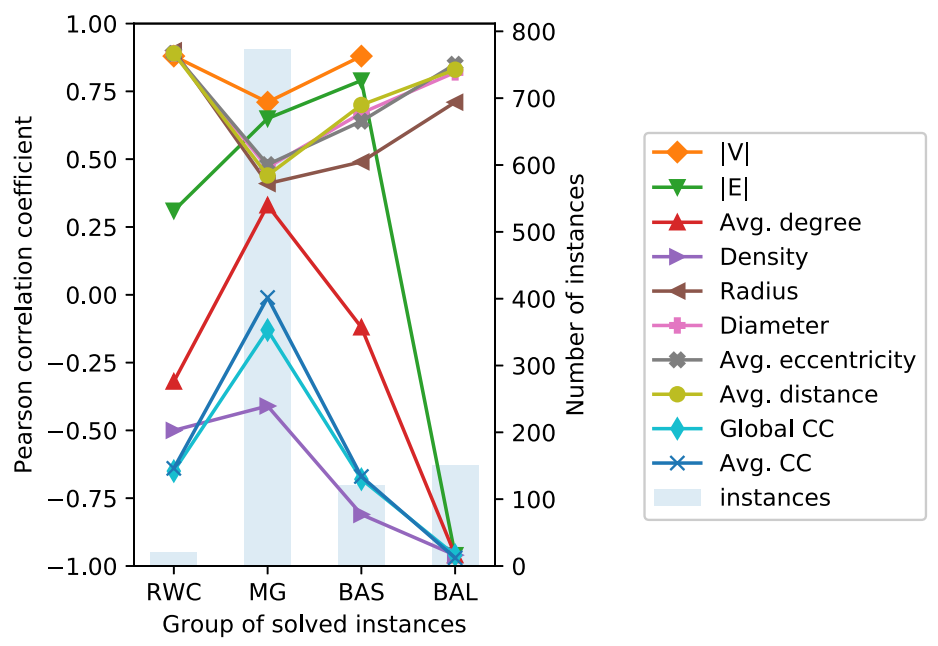

Figure 6. Pearson correlation coefficient between the optimal size OPT and some graph characteristics on the instances solved by at least one exact algorithm.

TABLE 5. Main quantitative information for each set of test instances exactly solved by algorithm EXACT-ENUM. Optimal solutions correspond to the number of distinct undirected longest induced paths. Distinct extremities account for the number of different extremities calculated over all optimal solutions.

\begin{tabular}{llllll}
\hline \hline & RWC & MG & BAS & BAL & Total \\
\hline Solved instances & 18 & 773 & 120 & 60 & 971 \\
Optimal solutions & 504 & 20859 & 1025 & 59292 & 81680 \\
Distinct extremities & 119 & 5523 & 619 & 732 & 6993 \\
\hline
\end{tabular}

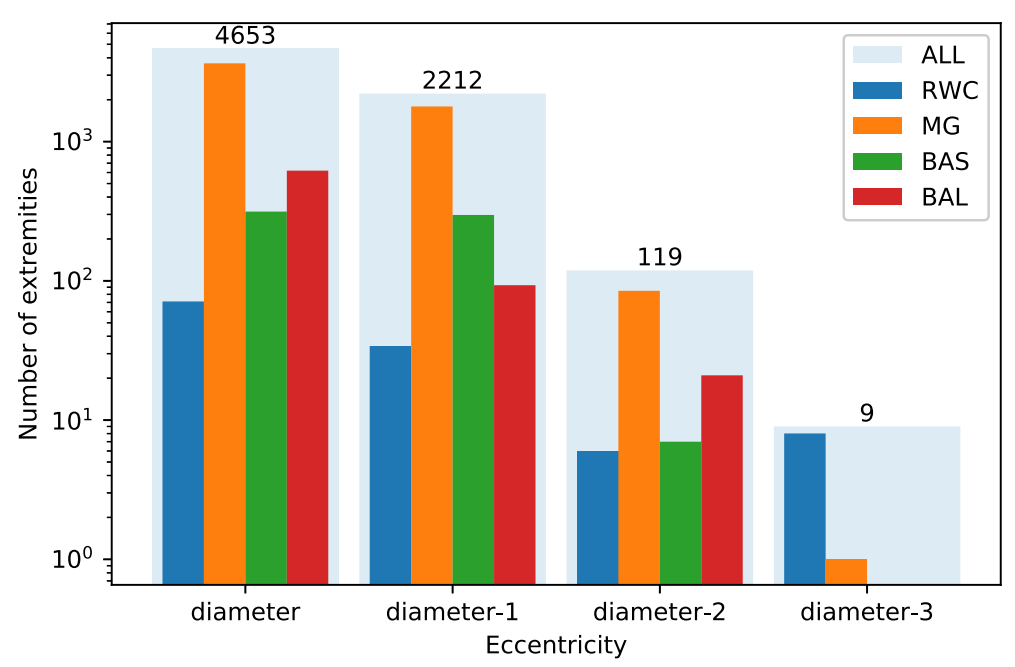

FIGURE 7. Number of extremities in the optimal paths vs. their eccentricities. 

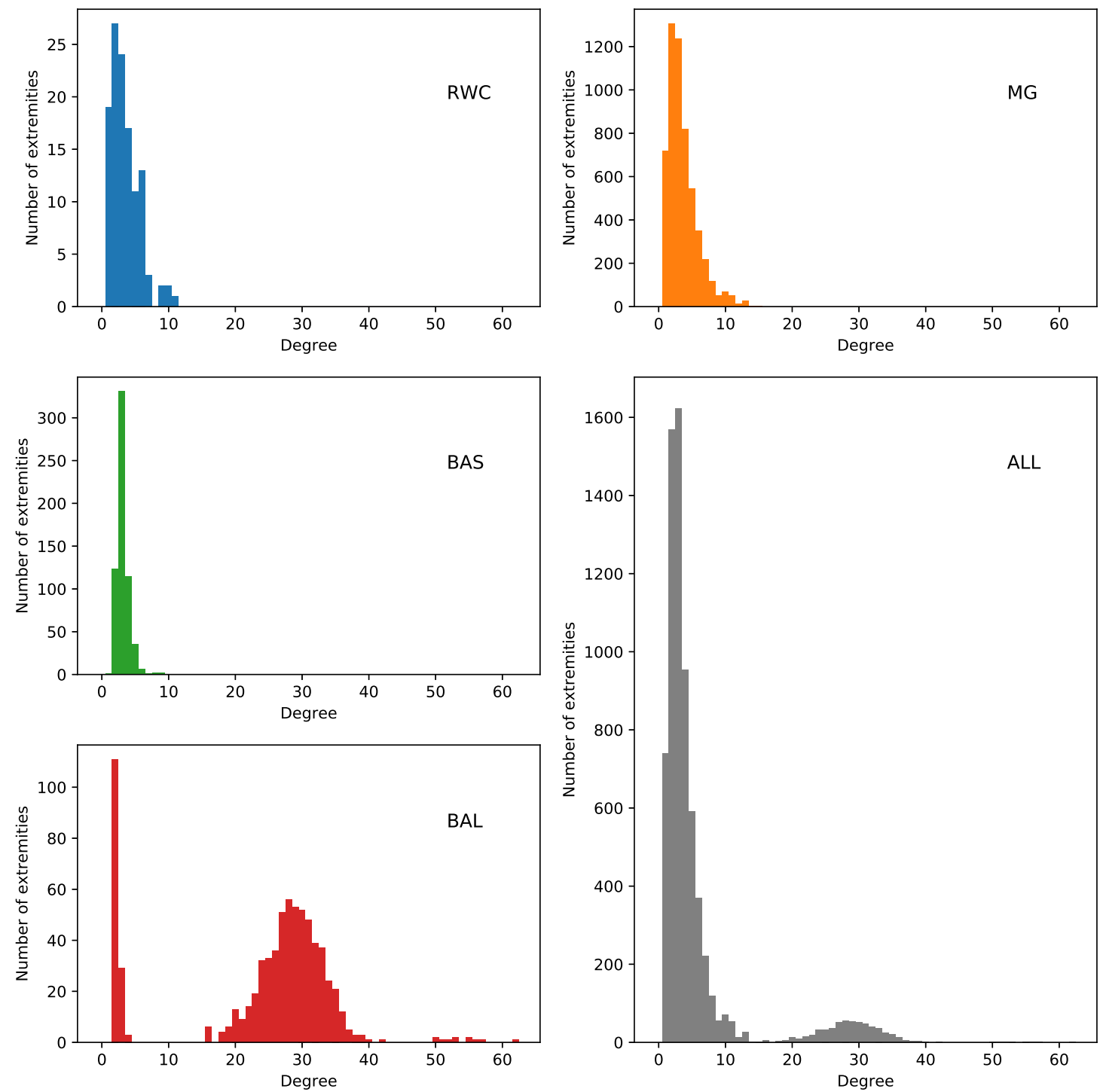

FIgURE 8. Number of extremities in the optimal paths vs. their degrees.

$C$ measures the cliquishness of a typical neighbourhood and is defined as follows. Suppose that a vertex $v$ has $k_{v}$ neighbours. Then, at most $k_{v}\left(k_{v}-1\right) / 2$ edges can exist between them. Let $C_{v}$ denote the fraction of these possible edges that actually exist. Define $C$ as the average of $C_{v}$ over all vertices $v$ of the graph. For friendship networks, this statistic has an intuitive meaning: $C_{v}$ reflects the extent to which friends of $v$ are also friends of each other and, consequently, $C$ measures the cliquishness of a typical friendship circle [20]. NetworkX [15] was used to compute all graph characteristics. Each set is represented as a category.

Figure 5 shows a high positive correlation between the running times and the size OPT of the longest induced paths (as already illustrated in Fig. 4), as well as between the running times and the number $|V|$ of vertices in the graph. Some characteristics, such as the number of edges $|E|$, are highly correlated with the running times for some sets of test instances but not for others, while others, such as the graph density, have a negative correlation with the running times for all test sets, with a value very close to zero for the MG instances. 

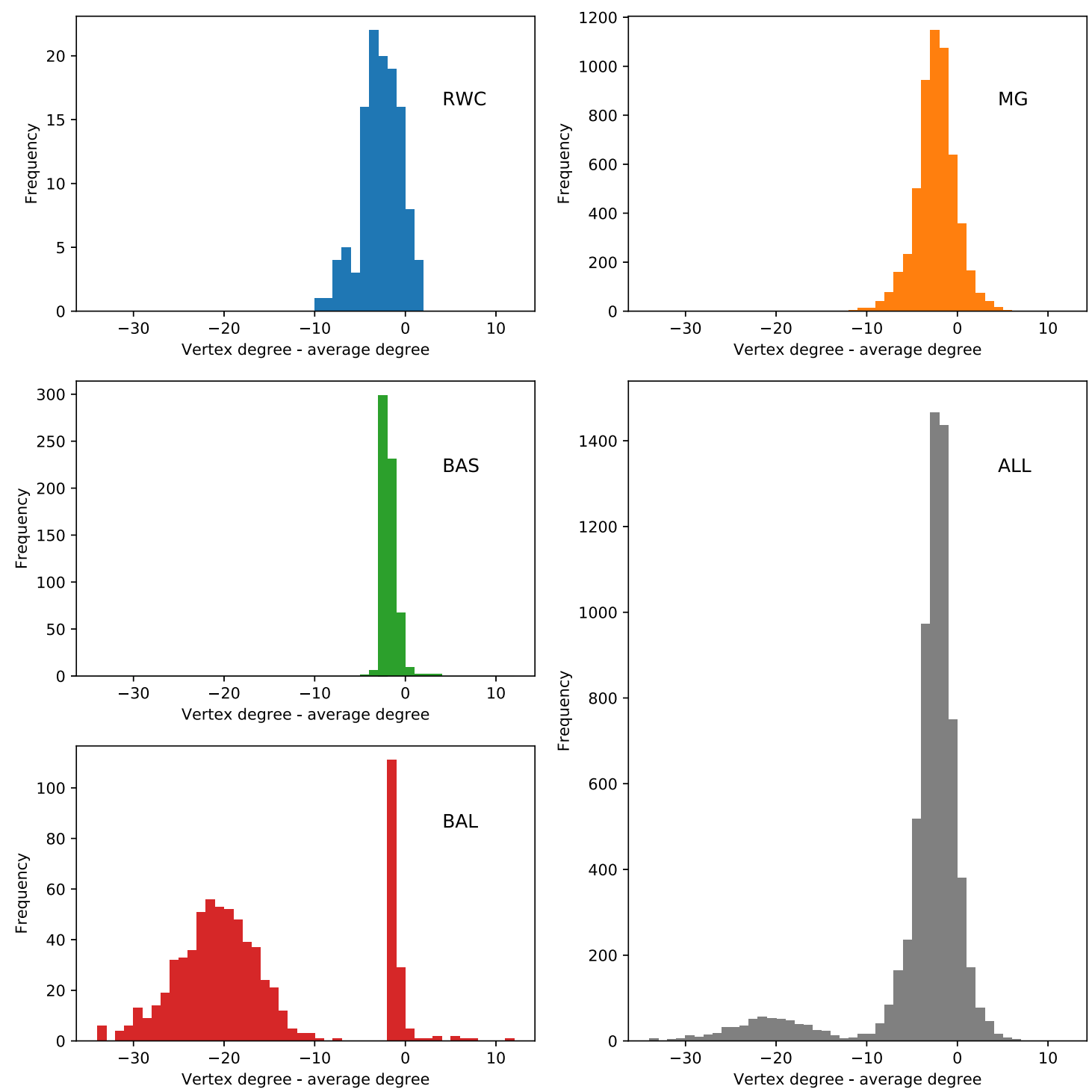

FiguRE 9. Frequency histogram of the degree differences of the extremities of the optimal solutions.

Figure 6 shows the Pearson correlation coefficient between the size OPT of the longest induced paths and some graph characteristics. It shows a high positive correlation between the size OPT of the longest induced paths and the number $|V|$ of vertices in the graph.

We also investigate the eccentricity and the degree of the extremities of the optimal paths obtained by our exact algorithm.

Table 5 summarizes the main quantitative information for each set of test instances exactly solved by algorithm EXACT-ENUM. We observe that although the 60 BAL instances represent only $6.2 \%$ of the total, they contribute with $72.6 \%$ of the optimal solutions found. On the other hand, the $773 \mathrm{MG}$ instances represent $79.6 \%$ of the total, but contribute with only $25.5 \%$ of the optimal solutions.

Figure 7 relates the number of extremities of the optimal paths with a diameter-based measure of their eccentricity. We provide the global information (considering all test instances exactly solved to optimality by 

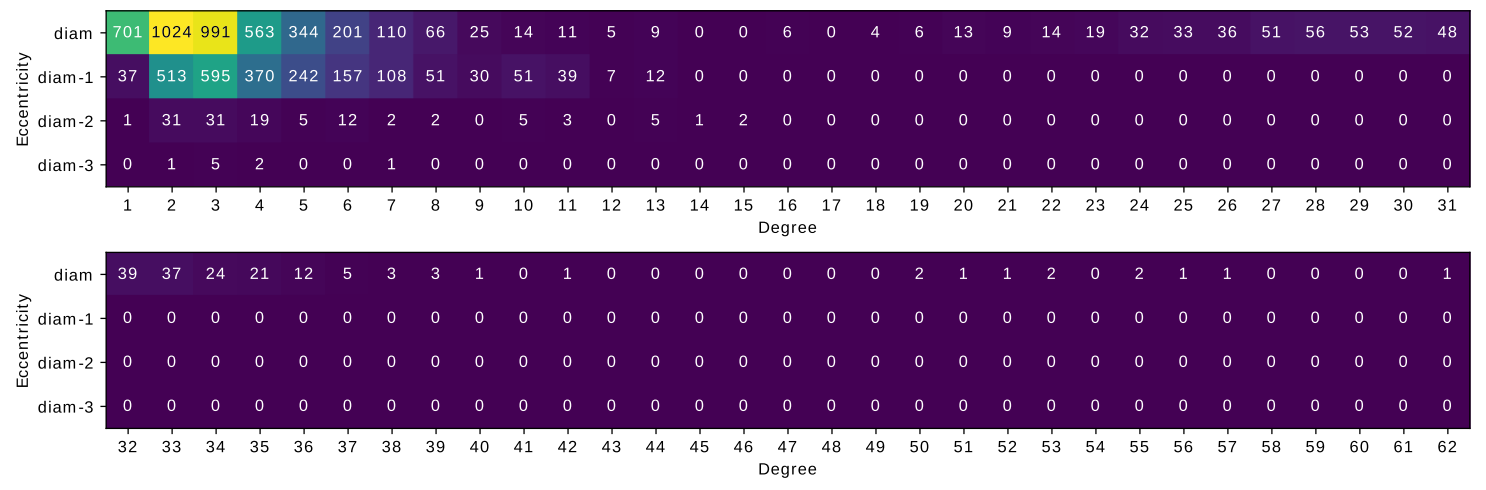

FiguRE 10. Number of extremities of optimal solutions with specific eccentricity and degree (over all instances solved by algorithm EXACT-ENUM).
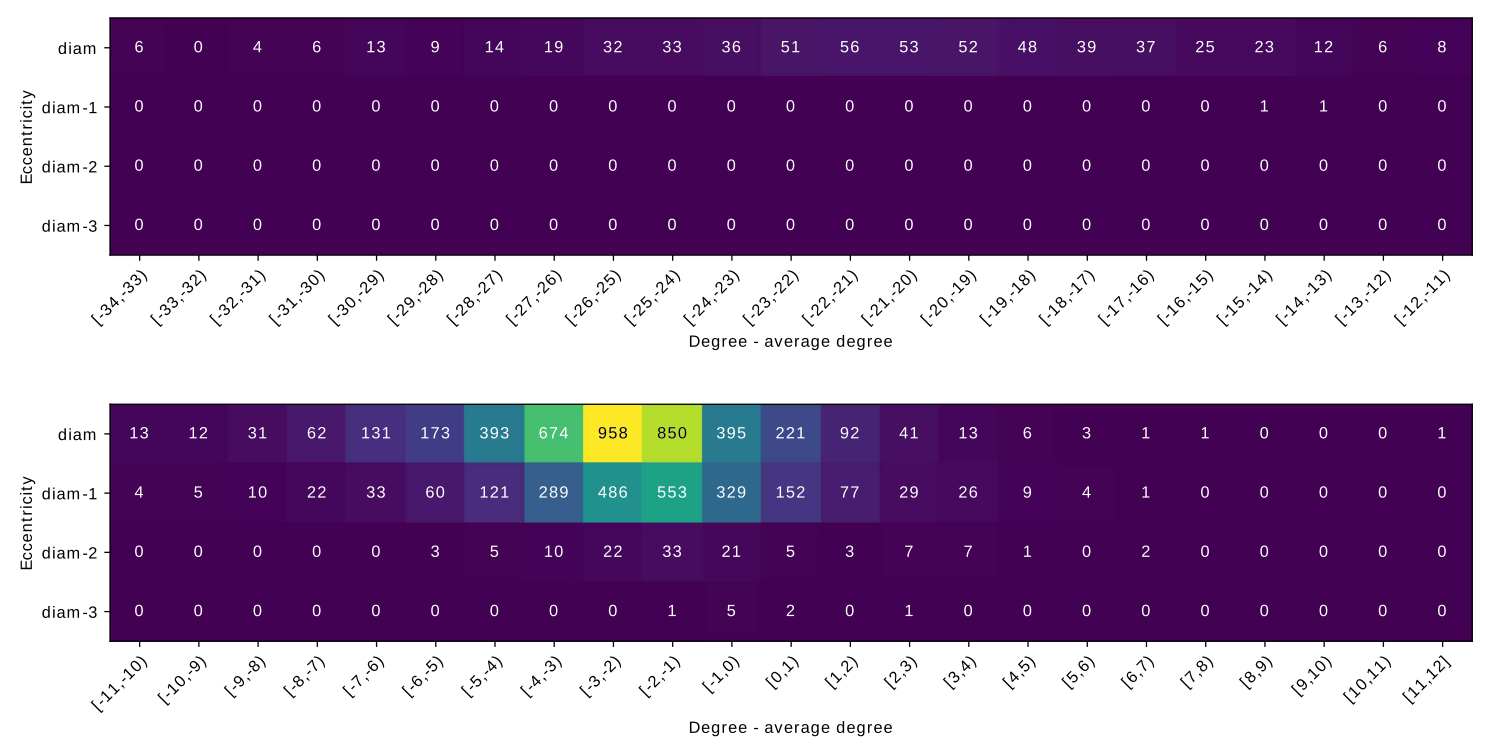

FigURE 11. Number of extremities of optimal solutions with specific eccentricity and interval of degree difference (over all instances solved by algorithm EXACT-ENUM).

algorithm EXACT-ENUM), as well as the information corresponding to each set of instances. We observe that $66.5 \%$ of the distinct extremities of the optimal paths have their eccentricities equal to the graph diameter, while $98.2 \%$ have their eccentricities greater than or equal to the diameter less one.

Figure 8 relates the number of extremities of the optimal solutions with their degrees. We provide the global information (considering all test instances exactly solved by algorithm EXACT-ENUM), as well as the information corresponding to each set of instances. We observe that $78.3 \%$ of the distinct extremities of the optimal paths have their degrees between 1 and 5, with $90.2 \%$ with degree less than or equal to 10. Extremities with degree greater than 15 (8.4\% of the total) appear only for instances in the BAL test set, which are those with the largest average degree.

We also evaluated the difference $D_{\text {deg }}$ between the degree of the extremities and the average degree. Figure 9 presents the frequency histogram of the degree difference (calculated as the extremity degree minus the average 
TABLE 6. Comparative time-to-target results between the greedy version G-HLIPP and heuristic HLIPP on large graphs.

\begin{tabular}{|c|c|c|c|c|c|c|c|c|c|c|c|c|}
\hline \multirow{3}{*}{ Name } & \multicolumn{3}{|c|}{ LH100 } & & & \multicolumn{2}{|c|}{ LH1000 } & & & \multicolumn{2}{|c|}{ LH10000 } & \\
\hline & & HLIPP & G-HLIPP & & & HLIPP & G-HLIPP & & & HLIPP & G-HLIPP & \\
\hline & Target & Time (s) & Time (s) & Ratio & Target & Time $(\mathrm{s})$ & Time (s) & Ratio & Target & Time $(\mathrm{s})$ & Time (s) & Ratio \\
\hline abyss & 6 & 0.0003 & 0.0002 & 0.715 & 6 & 0.0003 & 0.0002 & 0.878 & 6 & 0.0003 & 0.0002 & 0.763 \\
\hline hi-tech & 13 & 0.0062 & 0.0019 & 0.309 & 13 & 0.0355 & 0.0079 & 0.221 & 13 & 0.0345 & 0.0071 & 0.206 \\
\hline karate & 9 & 0.0118 & 0.0019 & 0.162 & 9 & 0.0132 & 0.0026 & 0.197 & 9 & 0.0131 & 0.0026 & 0.201 \\
\hline mexican & 16 & 0.0059 & 0.0029 & 0.488 & 16 & 0.0585 & 0.0107 & 0.183 & 16 & 0.1501 & 0.0109 & 0.072 \\
\hline sawmill & 18 & 0.0200 & 0.0087 & 0.437 & 18 & 0.0026 & 0.0026 & 0.995 & 18 & 0.0026 & 0.0022 & 0.865 \\
\hline pulp fiction & 7 & 0.0130 & 0.0014 & 0.109 & 7 & 0.0119 & 0.0014 & 0.118 & 7 & 0.0116 & 0.0013 & 0.114 \\
\hline chesapeake & 16 & 0.0231 & 0.0208 & 0.901 & 16 & 0.2175 & 0.1389 & 0.639 & 16 & 0.1154 & 0.0694 & 0.602 \\
\hline tailorS1 & 13 & 0.0011 & 0.0040 & 3.719 & 13 & 0.0011 & 0.0026 & 2.315 & 13 & 0.0010 & 0.0024 & 2.394 \\
\hline tailorS2 & 12 & 0.0030 & 0.0050 & 1.657 & 13 & 0.0179 & 0.0384 & 2.152 & 15 & 0.1668 & 0.1431 & 0.858 \\
\hline romeo and juliet & 9 & 0.0127 & 0.0083 & 0.653 & 9 & 0.0099 & 0.0027 & 0.275 & 9 & 0.0100 & 0.0032 & 0.319 \\
\hline oceans 12 & 8 & 0.0038 & 0.0053 & 1.400 & 8 & 0.0089 & 0.0069 & 0.771 & 8 & 0.0092 & 0.0070 & 0.761 \\
\hline die hard & 10 & 0.0046 & 0.0117 & 2.549 & 10 & 0.0061 & 0.0114 & 1.875 & 10 & 0.0064 & 0.0123 & 1.929 \\
\hline star wars II & 8 & 0.0086 & 0.0001 & 0.009 & 8 & 0.0143 & 0.0001 & 0.005 & 8 & 0.0152 & 0.0001 & 0.005 \\
\hline oceans 11 & 8 & 0.0061 & 0.0002 & 0.033 & 9 & 0.0338 & 0.0130 & 0.386 & 9 & 0.0349 & 0.0127 & 0.364 \\
\hline the departed & 8 & 0.0059 & 0.0014 & 0.245 & 8 & 0.0072 & 0.0016 & 0.220 & 8 & 0.0076 & 0.0016 & 0.206 \\
\hline krebs & 15 & 0.0171 & 0.0422 & 2.462 & 17 & 0.2056 & 0.3427 & 1.667 & 17 & 0.2374 & 0.4654 & 1.960 \\
\hline philadelphia & 9 & 0.0029 & 0.0073 & 2.478 & 10 & 0.0205 & 0.0837 & 4.079 & 10 & 0.0210 & 0.0812 & 3.871 \\
\hline 2012 & 11 & 0.0674 & 0.0550 & 0.817 & 12 & 0.0991 & 0.0161 & 0.163 & 12 & 0.1091 & 0.0165 & 0.151 \\
\hline braveheart & 11 & 0.0003 & 0.0077 & 28.657 & 11 & 0.0003 & 0.0365 & 135.056 & 11 & 0.0003 & 0.0362 & 121.688 \\
\hline huck & 9 & 0.0766 & 0.0175 & 0.229 & 9 & 0.0106 & 0.0119 & 1.122 & 9 & 0.0099 & 0.0122 & 1.232 \\
\hline gandhi & 9 & 0.0605 & 0.0026 & 0.042 & 10 & 0.0356 & 0.0468 & 1.314 & 10 & 0.0371 & 0.0439 & 1.182 \\
\hline watchmen & 9 & 0.0218 & 0.0002 & 0.008 & 9 & 0.0483 & 0.0002 & 0.004 & 9 & 0.0465 & 0.0002 & 0.004 \\
\hline jean & 11 & 0.0734 & 0.0047 & 0.063 & 11 & 0.2862 & 0.0064 & 0.022 & 11 & 0.3983 & 0.0062 & 0.016 \\
\hline godfather II & 16 & 0.1159 & 0.0014 & 0.012 & 18 & 0.0194 & - & - & 18 & 0.0188 & 0.5736 & 30.586 \\
\hline catch me if you can & 8 & 0.0014 & 0.0139 & 10.186 & 8 & 0.0013 & 0.0225 & 16.874 & 8 & 0.0011 & 0.0225 & 19.719 \\
\hline david & 18 & 0.0037 & 0.1002 & 27.039 & 19 & 0.8180 & 0.2282 & 0.279 & 19 & 1.3102 & 0.5103 & 0.390 \\
\hline doors & 12 & 0.2704 & 0.0078 & 0.029 & 12 & 0.0367 & 0.0363 & 0.989 & 12 & 0.0361 & 0.0356 & 0.984 \\
\hline public enemies & 17 & 0.0254 & 0.0150 & 0.589 & 20 & 0.1028 & 0.1097 & 1.067 & 20 & 0.1581 & 0.2191 & 1.386 \\
\hline santafe & 13 & 0.0268 & 0.0003 & 0.010 & 13 & 0.0318 & 0.0003 & 0.009 & 13 & 0.0321 & 0.0003 & 0.009 \\
\hline anna & 16 & 0.3898 & 0.0916 & 0.235 & 20 & 2.1087 & 0.1930 & 0.092 & 20 & 5.0350 & 0.4931 & 0.098 \\
\hline attiro & 28 & 0.0827 & 0.0699 & 0.846 & 29 & 0.2321 & 0.0166 & 0.072 & 30 & 1.8867 & 2.9333 & 1.555 \\
\hline dolphins & 20 & 0.0106 & 0.0013 & 0.124 & 22 & 0.8837 & 0.2206 & 0.250 & 23 & 0.0866 & 0.7646 & 8.827 \\
\hline prison & 28 & 0.0795 & 0.0161 & 0.202 & 31 & 0.7005 & 0.0834 & 0.119 & 36 & 1.9405 & 0.0460 & 0.024 \\
\hline sanjuansur & 35 & 0.0832 & 0.0179 & 0.216 & 35 & 0.6871 & 0.1091 & 0.159 & 37 & 11.7761 & 4.0833 & 0.347 \\
\hline ieeebus & 44 & 0.2010 & 0.2563 & 1.275 & 44 & 1.6414 & 2.2618 & 1.378 & 47 & 4.4927 & 1.8234 & 0.406 \\
\hline USAir97 & 29 & 0.1148 & 0.0393 & 0.343 & 33 & 4.3122 & 2.4855 & 0.576 & 38 & 110.4310 & 169.3410 & 1.533 \\
\hline 494_bus & 101 & 1.3763 & - & - & 107 & 12.2402 & 6.2169 & 0.508 & 114 & 332.5640 & 7.3518 & 0.022 \\
\hline 662_bus & 242 & 15.3271 & 21.9074 & 1.429 & 242 & 94.1874 & 141.2080 & 1.499 & 242 & 887.9110 & 1332.9700 & 1.501 \\
\hline S.Cerevisae & 144 & 0.6410 & 30.0306 & 46.849 & 149 & 152.3800 & 30.9581 & 0.203 & 155 & 1806.8500 & 2133.2800 & 1.181 \\
\hline Number of times $G$ & HLIPP & was faster & than HLIPP & $26 / 39$ & & & & $26 / 39$ & & & & $24 / 39$ \\
\hline
\end{tabular}

degree), for each set of test instances and for all solved instances. Comparing Figures 8 and 9 for the complete set of instances, we notice that in the second there is a reduction in the range of values represented in the horizontal axis with a frequency greater than zero. This might be useful in a heuristic to cut the subset of vertices that will be explored as extreme vertices for optimal solutions. We observe that $73.6 \%$ of the distinct extremities of optimal solutions have $D_{\operatorname{deg}} \in[-5,0)$, with $88.4 \%$ of them with $D_{\operatorname{deg}} \in[-8 f, 2)$. Furthermore, $89.9 \%$ of the extremities have their degrees smaller than the average degree.

Considering all test instances solved by algorithm EXACT-ENUM, Figure 10 shows a heatmap representing the number of extremities of optimal solutions with specific eccentricity and degree. We notice that $51.8 \%$ of the distinct extremities of optimal solutions have their eccentricity equal to the graph diameter and degree less than or equal to five, with $88.6 \%$ with their eccentricity greater than or equal to the diameter less one and degree less than or equal to ten.

Similarly, for the same test instances solved by algorithm EXACT-ENUM, Figure 11 shows a heatmap representing the number of extremities of optimal solutions with specific eccentricity and interval of degree 
difference. The degree difference was calculated as the vertex degree less the average degree of the graph. In this case, we observe that $46.8 \%$ of the distinct extremities of optimal solutions have their eccentricity equal to the graph diameter and $D_{\text {deg }} \in[-5,0)$, with $86.8 \%$ with their eccentricity greater than or equal to the diameter less one and $D_{\operatorname{deg}} \in[-8,2)$.

These two heatmaps clearly indicate that both the eccentricity and the degree play an important role to characterize the extremities of optimal solutions to the longest induced path problem.

These results and investigations are useful to guide the search for optimal solutions and better algorithms. An improved greedy version G-HLIPP of Algorithm 3 was developed in which vertices are selected in the non-increasing order of their eccentricities. Ties are broken in favor of the vertex with smaller degree.

Table 6 compares the two heuristics HLIPP and G-HLIPP in terms of the time they take to find a target solution value. The targets are the best values obtained by heuristic HLIPP in Table 3. For each stopping criterion (i.e., LH100, LH1000, and LH10000), we indicate the ratio between the times taken by G-HLIPP and HLIPP. Ratios smaller (resp. greater) than one correspond to instances where the greedy heuristic G-HLIPP was faster (resp. slower) than HLIPP. We observe that this simple improvement reduced the times to target values for $65 \%$ of the runs (76 out of 117). The average time-to-target reduction between G-HLIPP and HLIPP for these runs was 0.315. On the other hand, for the remaining runs where HLIPP was still faster, the average time-to-target reduction between HLIPP and G-HLIPP was 0.459. We observe that for two runs G-HLIPP did not find the target before the stopping criterion.

\section{Concluding Remarks}

In this article, we first proposed an exact enumerative algorithm based on backtracking for the problem of finding the longest induced path in a graph. The new algorithm is faster than that of Matsypura et al. [13] and competitive with the best ILP $_{\text {Cut }}$ implementations of Bökler et al. [5].

We also developed a new heuristic for the problem, which explores all vertices of the graph as possible source vertices of induced paths. Computational results showed that the newly proposed heuristic was consistently faster and more robust than the randomized heuristic of Matsypura et al. [13] on the same test problems.

The computational experiments also presented a numerical study correlating the eccentricity and the degree of the vertices with the frequency in which they appear as extremities of optimal solutions, i.e., longest induced paths. These results lead to the implementation of an improved version of the heuristic, in which the vertices are explored in the non-increasing order of their eccentricities.

Acknowledgements. The authors are grateful to Mark Newman for sending the dataset of the Santa Fe Institute collaboration network. Work of Ruslán G. Marzo was supported by a CAPES scholarship and by FAPERJ scholarship E-26/200.330/2020. Work of Celso C. Ribeiro was partially supported by CNPq research grants 303958/2015-4 and 425778/2016-9 and by FAPERJ research grant E-26/202.854/2017. This work was also partially sponsored by Coordenação de Aperfeioamento de Pessoal de Nível Superior (CAPES), under Finance Code 001.

\section{REFERENCES}

[1] R.K. Ahuja, T.L. Magnanti and J.B. Orlin, Network flows. Elsevier (1988).

[2] P. Alles and S. Poljak, Long induced paths and cycles in Kneser graphs. Graphs Comb. 5 (1989) 303-306.

[3] A.-L. Barabási and R. Albert, Emergence of scaling in random networks. Science 286 (1999) 509-512.

[4] B. Bergougnoux and M.M. Kanté, A meta-algorithm for solving connectivity and acyclicity constraints on locally checkable properties parameterized by graph width measures. Preprint arXiv:1805.11275 (2018).

[5] F. Bökler, M. Chimani, M.H. Wagner and T. Wiedera, An experimental study of ILP formulations for the longest induced path problem. In Combinatorial Optimization, edited by M. Baïou, B. Gendron, O. Günlük and A.R. Mahjoub. In Vol. 12176 of Lecture Notes in Computer Science. Springer, Cham (2020).

[6] M.R. Garey and D.S. Johnson, Computers and Intractability: A Guide to the Theory of NP-Completeness. Freeman and Co., New York (1979).

[7] F. Gavril, Algorithms for maximum weight induced paths. Inf. Process. Lett. 81 (2002) 203-208. 
[8] T. Ishizeki, Y. Otachi and K. Yamazaki, An improved algorithm for the longest induced path problem on k-chordal graphs. Discrete Appl. Math. 156 (2008) 3057-3059.

[9] L. Jaffke, O.-J. Kwon and J. Telle, Polynomial-time algorithms for the Longest Induced Path and Induced Disjoint Paths problems on graphs of bounded mim-width. In: Vol. 89 of Leibniz International Proceedings in Informatics (2018) 1-21.

[10] L. Jaffke, O.-J. Kwon and J.A. Telle, Mim-Width I. Induced path problems. Discrete Appl. Math. 278 (2020) $153-168$.

[11] W.H. Kautz, Unit-Distance Error-Checking Codes. IRE Trans. Electron. Comput. 7 (1958) 179-180.

[12] D. Kratsch, H. Müller and I. Todinca, Feedback vertex set and longest induced path on AT-free graphs. In: International Workshop on Graph-Theoretic Concepts in Computer Science. Springer (2003) 309-321.

[13] D. Matsypura, A. Veremyev, O.A. Prokopyev and E.L. Pasiliao, On exact solution approaches for the longest induced path problem. Eur. J. Oper. Res. 278 (2019) 546-562.

[14] Moviegalaxies, Social networks in movies. Available from: https://moviegalaxies.com/ (2020).

[15] NetworkX developers, NetworkX documentation. Available from: http://networkx.github.io/ (2020).

[16] M.E. Newman, The structure and function of complex networks. SIAM Rev. 45 (2003) 167-256.

[17] PassMark, PassMark Software - CPU Benchmarks. Intel Core i5-4460S vs. Intel Xeon E5-1650 v2 vs. Intel Xeon Gold 6134. Available from: https://www. cpubenchmark. net/compare/Intel-i5-4460S-vs-Intel-Xeon-E5-1650-v2-vs-Intel-XeonGold-6134/2232vs2066vs3008 (2020).

[18] K. Pearson, Notes on regression and inheritance in the case of two parents. Proc. R. Soc. London 58 (1895) $240-242$.

[19] L.O. Prokhorenkova and E. Samosvat, Global clustering coefficient in scale-free networks. In: International Workshop on Algorithms and Models for the Web-Graph. Springer (2014) 47-58.

[20] D.J. Watts and S.H. Strogatz, Collective dynamics of "small-world" networks. Nature 393 (1998) 440-442. 